University of Rhode Island

DigitalCommons@URI

Open Access Master's Theses

2016

Novel Catalyst Development for Chemical Sensors

Zachary Caron

University of Rhode Island, zcaron8@gmail.com

Follow this and additional works at: https://digitalcommons.uri.edu/theses

Recommended Citation

Caron, Zachary, "Novel Catalyst Development for Chemical Sensors" (2016). Open Access Master's

Theses. Paper 846.

https://digitalcommons.uri.edu/theses/846

This Thesis is brought to you for free and open access by DigitalCommons@URI. It has been accepted for inclusion in Open Access Master's Theses by an authorized administrator of DigitalCommons@URI. For more information, please contact digitalcommons-group@uri.edu. 
NOVEL CATALYST DEVELOPMENT FOR CHEMICAL

SENSORS

BY

ZACHARY CARON

A THESIS SUBMITTED IN PARTIAL FULFILLMENT OF THE

REQUIREMENTS FOR THE DEGREE OF

MASTER OF SCIENCE

IN

CHEMICAL ENGINEERING

UNIVERSITY OF RHODE ISLAND

2016 


\section{MASTER OF SCIENCE THESIS}

OF

ZACHARY CARON

\section{APPROVED:}

Thesis Committee:

Major Professor $\quad$ Otto Gregory
Everett Crisman
Tao Wei
Nasser H. Zawia
DEAN OF THE GRADUATE SCHOOL

UNIVERSITY OF RHODE ISLAND

2016 


\begin{abstract}
In recent years, the use of improvised explosive devices (IED's) has been the preferred method for terrorist attacks because of their practical development from available resources. From the "Shoe Bomber" attack back in 2001, to the most recent attacks in Paris, IED's have been a vehicle for explosive delivery and of the utmost concern for organizations such as the Department of Homeland Security. Conventional methods for the detection of explosives have resorted to either canines, also known as "drug-sniffing” dogs, or the use of ion-mobility spectrometry. While such methods have proven some success in the field in the past, they are invasive, costly, or most importantly non-continuous. With this in mind, a continuous system for the detection of explosives at trace levels has been developed here at the University of Rhode Island.

Recent improvements in the electronic trace detection system have included the use of pre-concentration in addition to the dynamic sensing mechanism. This allows for a controlled and highly concentrated burst of analyte to be delivered to the thermodynamic sensor. With the ultimate goal to fabricate a MEM's based device, a reduction in thermal mass without sacrificing signal response is imperative.

To combat the reduction in thermal mass, metal oxide nanowires have been developed to create a library of catalysts that can be used to specifically target explosive molecules. Metal oxide nanowires increase viable sensor surface area by orders of magnitude, which is necessary for the transition to a MEMS based device. Not only has the surface area been increased, but the minimum
\end{abstract}


detection limits for explosives such as 2, 6-DNT have been demonstrated to be improved down to the ppb and ppt levels.

Finally, a portable testing setup has been developed which will allow for field-testing and generation of real-time results. By reducing the sensor footprint and reconfiguring the apparatus so that it fits into a carrying case, we will be able to test for explosives in a number of different environments. 


\section{ACKNOWLEDGEMENTS}

I would first like to thank Dr. Otto J. Gregory for his continued guidance and support throughout my tenure here at the University of Rhode Island. It has been an honor to learn from an expert in the field of sensors and I could not have wished for any more in my experience here.

I would also like to thank a number of fellow students who have helped to make this research the success that it is today. At the beginning of this project, Daniel Mallin was an integral part of providing me with a strong background and basis for my research. Mitch Champlin also played a major role in the transition of the project over to me. Lastly, I would like to thank Vivek Patel. Without his hard work and determination, the research would not be where it is today.

I would like to express gratitude towards my committee members as well, Dr. Everett Crisman, Dr. Tao Wei, and Dr. Alan Davis. Their expertise has helped me to take this project to where it is today and I have attained a wealth of knowledge from each of them.

I would like to give a very special thanks for Mr. Michael Platek. He has been an amazing person to work with in my tenure here and ultimately a great mentor. By challenging me every day and teaching me skills outside the literature searches, I feel better prepared to be successful in the field of chemical engineering and I thank you sincerely for that.

Lastly, I would like to thank my family and friends. Without their continued support throughout my studies, I would not be where I am today. 


\section{TABLE OF CONTENTS}

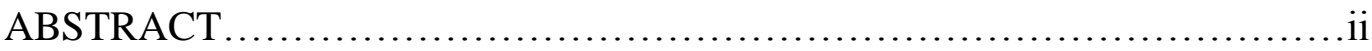

ACKNOWLEDGMENTS ...................................................iv

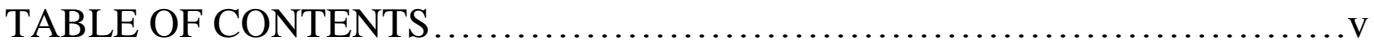

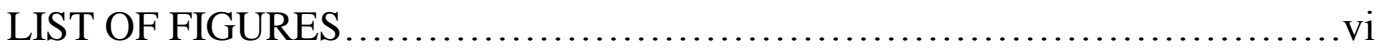

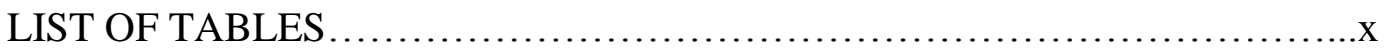

CHAPTER 1 - INTRODUCTION.........................................

CHAPTER 2 - LITERATURE REVIEW ...................................

2.1 Introduction to Explosives.............................................

2.1.1 Review of Common Explosives...................................5

2.1.2 Explosive Compounds used in IEDs.............................

2.2 Explosive Detection Technologies....................................10

2.2.1 Bulk Detection Technology ...................................11

2.2.2 Trace Detection Technology..................................13

2.2.3 Dr. Otto Gregory SSTP Laboratory Group Accomplishments.......18

2.2.4 Thermodynamic Sensing Improvements.........................21

CHAPTER 3 - METHODOLOGY ..........................................27

3.1 Thermodynamic Gas Sensor..........................................27

3.1.1 Nickel Microheater Patterning................................28

3.1.2 Passivation Layer Fabrication....................................29

3.1.3 Metal Oxide Nanowire Catalysts...............................30

3.2 Dynamic Sensor Testing ............................................33

3.2.1 Testing Techniques using the Dynamic Approach...................34 
CHAPTER 4 - RESULTS ............................................. 38

4.1 Metal Oxide Nanowire Catalysts......................................38

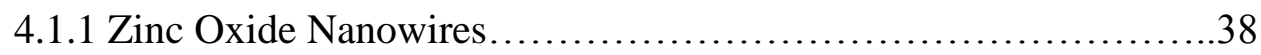

4.1.2 Copper Oxide Nanowire Catalyst..............................46

4.1.3 Iron Oxide Nanowire Catalyst...................................49

4.2 Sensor Design that Targets Explosives...................................54

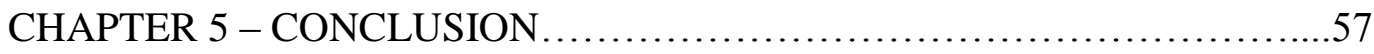

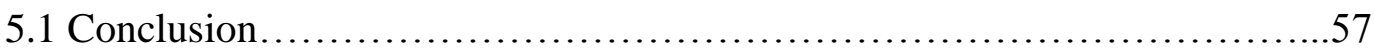

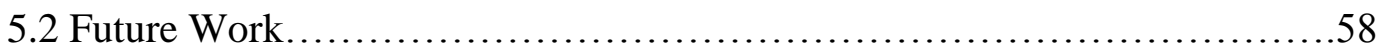

5.2.1 Conductometric Sensor Applications.............................59

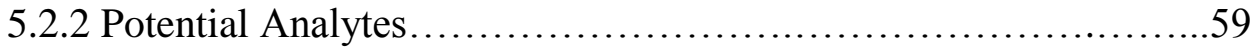

5.2.3 Transition towards Environmental Testing.......................60

5.2.4 Pre-concentration Improvement..................................61

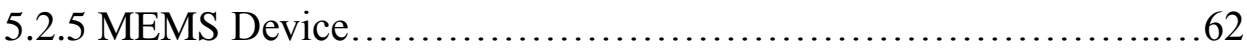

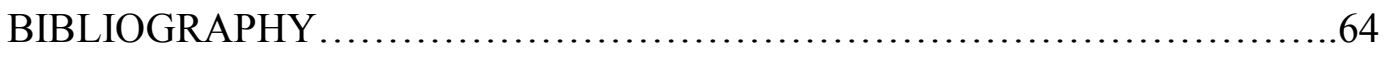




\section{LIST OF FIGURES}

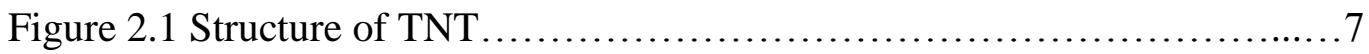

Figure 2.2 Structure of 2,6 DNT ......................................... 8

Figure 2.3 Structure of Ammonium Nitrate ....................................9

Figure 2.4 Structure of triacetone-triperoxide (TATP) $\ldots \ldots \ldots \ldots \ldots \ldots \ldots \ldots \ldots . . . \ldots$

Figure 2.5 Flow chart for explosive detection...............................10

Figure 2.6 Commercially available X-ray system for bulk detection (left) with a potentially dangerous IED outlined in red using X-ray technology (right).

Figure 2.7 CT Scan showing the image of a piece of luggage (above) with the various cross-sectional images taken (below) and a potential threat highlighted in

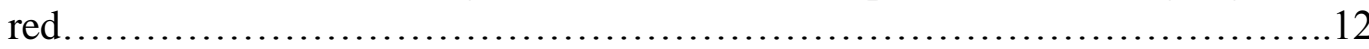

Figure 2.8 Backscatter image (left) of a suitcase with four potential threats identified while hidden within a radio which was not identified by the transmission x-rays (right) .................................................

Figure 2.9 Portable GC/MS detection system used for particulate detection......14

Figure 2.10 Components ofIMS particulate detection system...................16

Figure 2.11 Concentration of some common explosives in air at room temperature........................................................ 17

Figure 2.12 Schematic of the trace detection system employing dynamic control................................................................. 19

Figure 2.13 Thermodynamic sensor response to TATP from both the catalyst coated sensor (blue) and the dynamic control (red)...........................20

Figure 2.14 Conductometric response to 2, 6-DNT employing a ZnO catalyst at

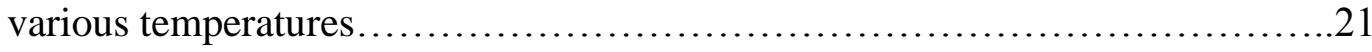

Figure 2.15 Schematic of pre-cocentration test bed for thermodynamic sensing

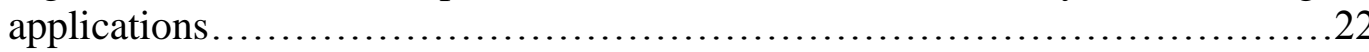

Figure 2.16 Preconcentrator duty cycle beginning with the introduction of 2, 6DNT vapor to the preconcentrator (1), followed by preconcentrator thermal 
desorption of DNT (2), and lastly the preconcentrator is turned off while reference

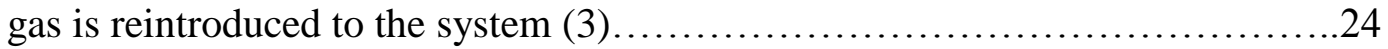

Figure 2.17 Schematic diagram of the MEMS based platform for chemical sensing.

Figure 3.1 Schematic of the various layers comprising the thermodynamic sensor. The alumina ceramic substrate (A), Ni microheater (B), aluminum oxide passivation layer $(\mathrm{C})$ and the metal oxide catalyst $(\mathrm{D})$.

Figure 3.2 Sensor responses to various concentrations of TATP showing the merit of using a porous alumina cement (blue) versus no cement (red). 30

Figure 3.3 Schematic of electronic detection system using dynamic control in conjunction with nanowire catalyst....

Figure 4.1 SEM micrographs depicting the various stages of zinc oxide nanowire development. 39

Figure 4.2 SEM micrographs comparing the sputter deposited zinc oxide film (left) to the high surface area zinc oxide nanowire catalyst (right)

Figure 4.3 Sensor components employing oxide nanowires as a catalyst support; alumina substrate (A), Ni microheater (B), alumina oxide passivation layer (C), zinc oxide nanowires (D), sputter deposited catalyst (E)

Figure 4.4 Response of two sensors with different surface area catalysts; a sputtered tin oxide catalyst (blue) and a nanowire catalyst support (red) with sputtered tin oxide catalyst, exposed to 2, 6-DNT.

Figure 4.5 Responses of two sensors exposed to TATP; one sensor was fabricated with a nanowire catalyst support (blue) and the other with a sputtered catalyst (red) only.

Figure 4.6 XRD pattern of zinc oxide nanowires formed on the sensor surface.

Figure 4.7 XPS spectra showing the $\mathrm{Zn} 2 \mathrm{p}^{3}$ zinc oxide peak (red) shifting to lower energy corresponding to zinc metal after 30 seconds of sputter etching (blue).

Figure 4.8 SEM micrograph of copper oxide nanowires directly grown on the thermodynamic sensor.

Figure 4.9 SEM micrograph of copper oxide nanowires thermally grown at 340C for 5hours. 
Figure 4.10 XRD pattern of the copper oxide nanowire catalyst................48

Figure 4.11 XPS analysis of the copper oxide nanowire catalyst...............49

Figure 4.12 SEM micrograph of iron oxide nanowires thermally grown on the serpentine of the microheater............................................ 50

Figure 4.13 SEM micrograph of iron oxide nanowire growth at 600C..........51

Figure 4.14 XRD pattern of the thermally grown iron oxide nanowires..........52

Figure 4.15 XPS overlay plot of iron oxide nanowires prior to and after sputter

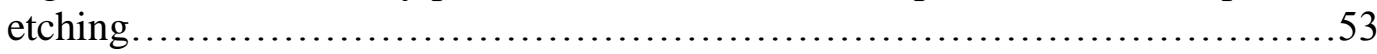

Figure 4.16 XPS analysis of the iron oxide nanowires......................53

Figure 4.17 Sensor response to TATP in the presence of methyl acrylate........54

Figure 5.1 Portable testing system for field applications....................60

Figure 5.2 Applications for metal oxide nanowire catalysts on MEMS based devices.......................................................63 


\section{LIST OF TABLES}

Table 2.1 Properties of polymer candidates for pre-concentrator applications.......................................................... 23

Table 5.1 Detection limits for threat molecules using zinc oxide nanowire catalyst

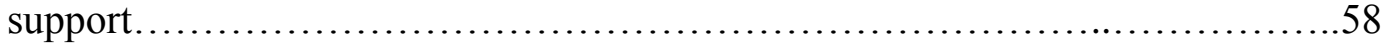




\section{CHAPTER 1}

\section{INTRODUCTION}

In recent years, the use of improvised explosive devices (IEDs) has been the preferred method by terrorists to carry out deadly attacks on civilians. Beginning as far back as 1993 with the truck bombings in New York city, and as recently as the Paris bombings in 2015, IEDs are becoming more relevant in that they can be devised from readily available materials, instill great fear in the travelling public, and ultimately cause great damage if not disarmed. Not only that, but innocent civilians are not the only targets of such an attack [1]. IEDs are used across the world in Afghanistan and Iraq as a means to defend against soldiers. As of 2013, more than $60 \%$ of all combat casualties from conflict in Iraq and Afghanistan were the result of IEDs [1]. That percentage results in nearly 3,200 killed and 33,000 wounded. In other various parts of the world, an average of 700 explosions per month are the result of an improvised explosive attack [2]. As these numbers continue to rise, and public becomes increasingly fearful, the need for a successful system for the detection of explosives is paramount.

While conventional methods for explosive detection, such as ion mobility spectrometry (IMS) or canines have merit in the field of explosive detection, they are hindered in some areas. On the one hand, IMS method requires a cotton swab of a person's clothing or luggage in order to test for explosives. This can become cumbersome for busy travelers. On the latter end, canines, although the gold 
standard for explosive detection, are not a continuous system in that they require periods of rest and possess a high sense of smell for limited years of their life. This being said, a robust detection system that can continuously monitor the air for these energetic compounds is necessary.

Over the past eight years, at the University of Rhode Island, an orthogonal sensor for the detection of explosives has been developed in an attempt to address this issue. The sensor would be comprised of both a conductometric and a thermodynamic element in an attempt to distinguish whether or not a given analyte is present in the vapor phase. By using what is considered to be an orthogonal platform, the sensor would have the ability to mitigate false positives by creating a unique signature for each explosive analyte [3]. Recently, the sensor has been able to detect high energy materials such as TATP in the parts per million range, but in order to move towards commercialization, lower detection limits for explosives needs to be achieved [3].

Thus, the primary goal of this research was to make key improvements to the thermodynamic sensor to achieve lower detection limits associated with energetic materials as well as create a portable testing system. This was achieved by developing a high surface area catalyst that would not only lower detection limits, but also contribute towards the transition to a Micro-Electro-Mechanical System (MEMS). The transition to a MEMS platform requires a major reduction in thermal mass without sacrificing catalytic interactions, which are necessary for detection. Metal oxide nanowire catalysts were developed to combat such a reduction in thermal mass. Zinc oxide, Copper oxide, and Iron oxide nanowires 
were fabricated and characterized using SEM, XRD, and XPS. Nanowire catalysts proved to provide not only additional viable surface area, but also to decrease detection limits for compounds such as 2, 6-DNT and TATP to the parts per billion level. 


\section{REFERENCES}

[1] Wilson, Clay. Improvised Explosive Devices (IEDs) in Iraq and Afghanistan: Effects and Countermeasures. (2007): 1-3. Federation of American

Scientists, 21 Nov. 2007. Web. 3 Sept. 2014.

[2] Barbero, Michael D. Improvised Explosive Devices Are Here to Stay. The

Washington Post. N.p., 18 May 2013. Web. 6 Sept. 2014.

[3] Mallin D. Increasing the Selectivity and Sensitivity of Gas Sensors for the

Detection of Explosives. University of Rhode Island M.S. thesis (2014). 


\section{CHAPTER 2}

\section{LITERATURE REVIEW}

\subsection{Introduction to Explosives}

As a working definition, explosives, can be defined as substances, which on initiation by friction, impact, shock, spark, flame, heating, or any simple application of an energy pulse, results in a rapid chemical reaction that releases heat, exerting an outward pressure on its surrounding[1]. There are a number of these compounds, also referred to as energetic materials, which exhibit varying degrees of danger based upon their chemical structures.

\subsubsection{Review of Common Explosives}

Since the focus of this research is on explosive detection, a review of the most common explosives is warranted, and most notably those used in the construction of IEDs. Generally speaking, explosives usually have chemical structures that include a carbon based backbone with either nitrogen-nitrogen bonds or nitrogen-oxygen bonds attached, which are considered oxidizing groups [1]. Upon an explosion, an internal redox reaction occurs that releases a gaseous product (either $\mathrm{N}_{2}$ or $\mathrm{CO}_{2}$ ) [1]. Concurrently, all of the original non-bonding electrons in the structure form stable pi-bonds.

One way that these explosives can be characterized, in terms of severity, is by how quickly and under what conditions these chemicals detonate. Using these guidelines, explosives can either be considered low explosives or high explosives. 
"Low explosives, which are also known as propellants, while still containing the oxygen needed for their combustion, are at most combustible materials which undergo deflagration by a mechanism of surface burning" [1]. These low energy explosives, which include gunpowder, smokeless powder and gun propellants, have the ability to detonate under confined conditions resulting from the pressure build up when undergoing chemical reactions. On the other hand, high explosives undergo the physical phenomenon of detonation because of their rapid chemical reactions [1]. As these chemical reactions go to completion, a high-pressure shock wave moves though the explosive. High explosives such as TNT, NG, RDX, HMX are defined by their velocity rates of detonation, which can range from $5500-9500 \mathrm{~m} / \mathrm{s}$, with the latter being the most destructive.

Energetic compounds can also be classified based on their sensitivity. Depending on a terrorist's plan of attack, they may not want to use a compound that is highly sensitive to outside stimuli, especially if they are attempting to transport the material. On the other hand, depending on their plan of attack, a terrorist may desire an explosion upon the slightest of interaction with the IED. Such compounds are referred to as secondary and primary explosives respectively. Primary explosives, which are also known as initiators, are utilized to detonate other explosives because of their relatively unstable nature when exposed to outside stimuli. They allow a user to detonate a larger explosion when necessary. These larger explosions are the result of secondary explosives. Contrary to the primary explosives, secondary explosives violently react with 
significant outside stimuli, usually the result of a shock or a high energy pulse from an initiator.

\subsubsection{Explosive Compounds used in IEDs}

As mentioned previously, IED refers to improvised explosive devices, and the fact that they are "improvised" means that they are being constructed from readily available materials. Some of the most commonly used chemicals in the development of IEDs include, but are not limited to, TNT, RDX, HMX, PETN, AN, and TATP, with a few of them discussed in the following sections.

TNT (trinitrotoluene) is one of the most widely used explosives in IEDs and is viewed as the standard for explosive compounds (figure 2.1).

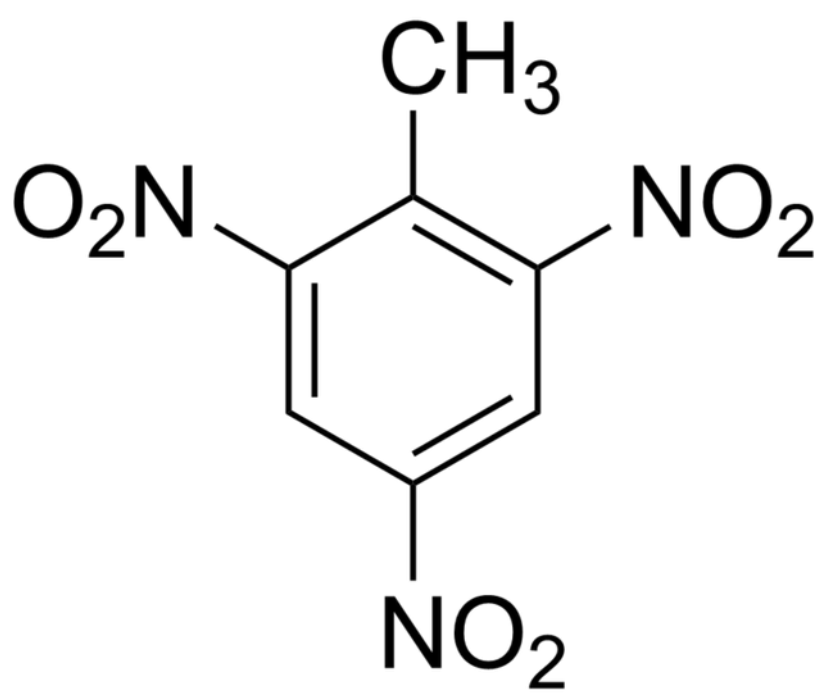

Figure 2.1 Structure of TNT

As a result of its relatively stable structure upon small impact, it has been used in military combat by being poured inside of certain bullet shells. The attractive feature of TNT is that its melting temperature (80C) is much lower than its detonation temperature. This allows the explosive to not only be mixed with additional chemicals, but also to be transported easily since it requires an energetic pulse to cause rapid oxidation. Rapid internal oxidation from the 
numerous oxygen groups are what cause this chemical to be so deadly. In addition, nitrogen can form triple bonds during the oxidation process which also contains a high amount of energy that is released upon high impact. For experimental purposes, 2, 6 DNT (dinitrotoluene) shown in figure 2.2, was used to emulate TNT.

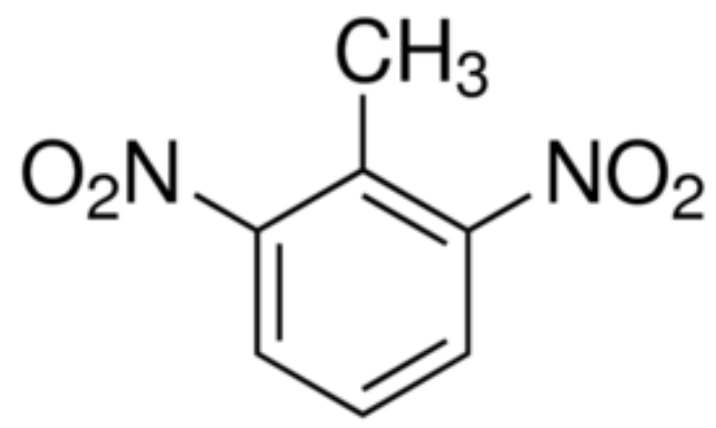

Figure 2.2 Structure of 2, 6 DNT

2, 6 DNT is similar to TNT with the exception of one fewer nitrogen dioxide group attached to the benzene ring. Having less nitrogen dioxide groups, 2, 6 DNT is slightly easier to work with in an experimental setting since the amount of internal oxidation is less severe than that of TNT. Because it is so similar in structure to TNT, 2, 6 DNT is a good measure for how successful the sensing device is.

Another nitrogen based explosive that is commonly used in IEDs is ammonium nitrate (figure 2.3). 


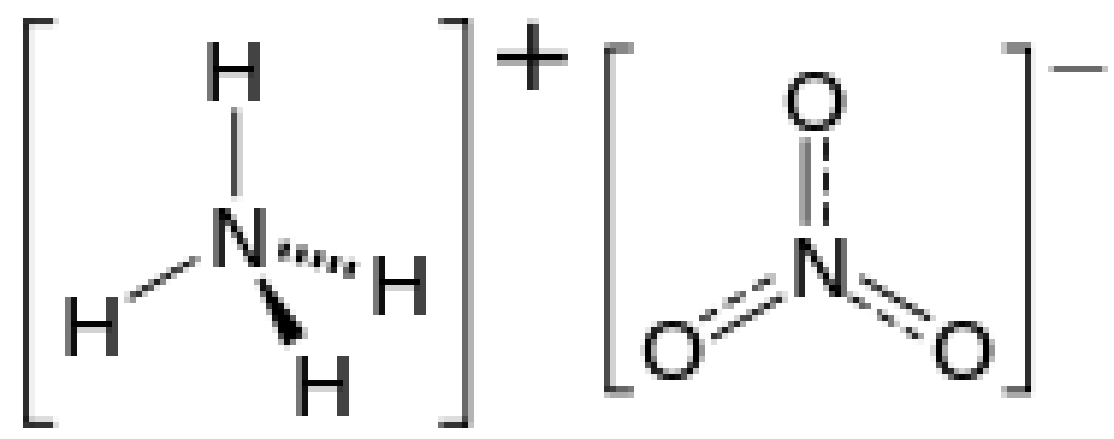

Figure 2.3 Structure of Ammonium Nitrate

Similar to that of both DNT and TNT, N-O bonds are realized which causes the chemical to be a strong oxidizer. It is commonly found in fertilizers which makes it an ideal candidate for use in IEDs. It is one of the main components of ANFO (ammonium nitrate fuel oil) and is used in combination with a booster (sticks of dynamite) that allow the explosive to fully release its energy and continue its detonation wave. Although it may be slightly more difficult to use than TNT because of hydrophilicity, its easy attainment makes it a highly touted chemical for terrorist attacks.

There are also those energetic materials that are non-nitrogen containing, one being TATP (tri-acetone-tri-peroxide) shown in figure 2.4.

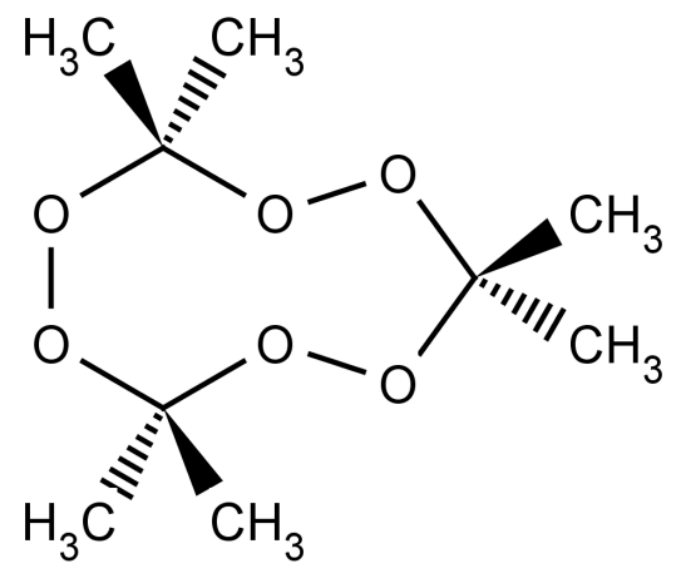

Figure 2.4 Structure of triacetone-triperoxide (TATP) 
Although TATP does not contain any nitrogen groups which form highly energetic bonds, it does contain many oxygen atoms making it a strong oxidizing agent. Since it only requires a simple reaction between hydrogen peroxide $\left(\mathrm{H}_{2} \mathrm{O}_{2}\right)$ and acetone $\left(\mathrm{C}_{3} \mathrm{H}_{6} \mathrm{O}\right)$, TATP has been used in many recent IEDs, most notably in the Paris 2015 attacks. It has also gained great publicity because it does not contain those nitrogen groups that most of severe explosives contain. Since most of the original detectors were developed with nitrogen-based explosives in mind, chemicals such as TATP were going undetected. Having the correct measures in place to scan for all of these various compounds is of the utmost important to prevent these attacks.

\subsection{Explosive Detection Techniques}

Explosive detection can be characterized in two different ways; (figure 2.5) bulk and trace detection. Bulk detection is the detection of the actual explosive, whereas trace detection detects either small particulates or vapors of the explosive material.

\section{Explosives Detection}

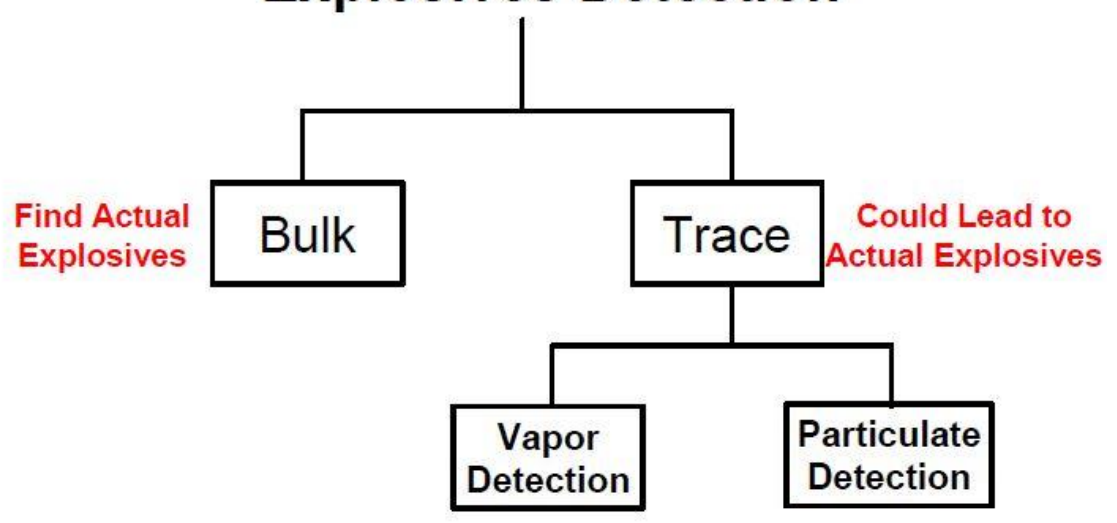

Figure 2.5 Flow chart for explosive detection 
As this research focuses on trace detection systems, a brief overview of bulk detection is warranted to provide a full picture.

\subsubsection{Bulk Detection Technologies}

There are a number of different technologies for bulk explosives detection including X-rays, CT Scans, and Backscatter electron imaging. Each method has its merits for preventing terrorist attacks by taking advantage of certain characteristics of typical compounds.

X-ray machines (figure 2.6) take advantage of chemical responses when exposed to X-rays. These responses can be in the form of either absorption, desorption, or scattering of rays.
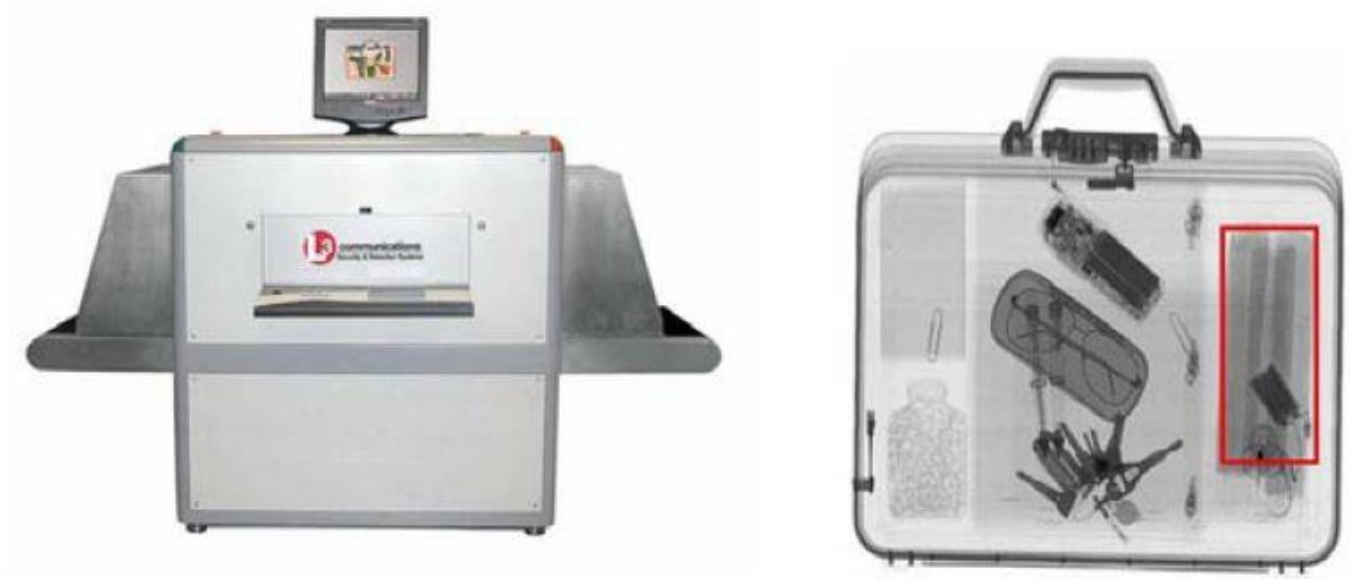

Figure 2.6 Commercially available X-ray system for bulk detection (left) with a potentially dangerous IED outlined in red using X-ray technology (right) ${ }^{1}$

Based on the absorption, transmission or scattering of such rays, object outlines are distinguishable which would alarm the user of a potentially hazardous IED. Although X-rays are powerful tools for viewing luggage from the outside,

\footnotetext{
${ }^{1}$ Photo furnished by L3 Communications Security and Detection Systems
} 
sometimes objects do not interact with X-rays and thus do not appear when screening takes place. In addition, X-ray machines do not have the ability to determine the thickness of an object and so the atomic number of a given substance becomes nearly impossible to determine.

Alternatively, CT Scans (figure 2.7) provide a much more in depth analysis of potential threats that are hidden inside baggage.

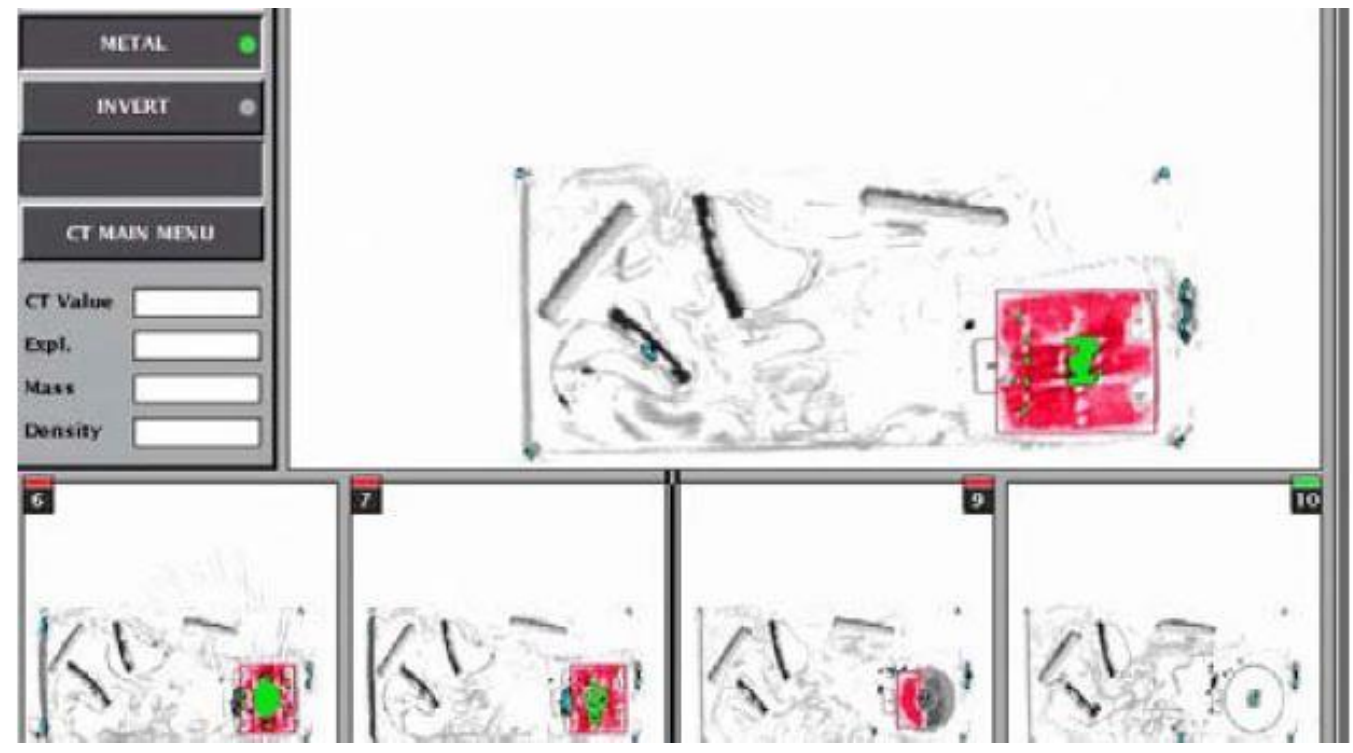

Figure 2.7 CT Scan showing the image of a piece of luggage (above) with the various crosssectional images taken (below) and a potential threat highlighted in red ${ }^{2}$

In CT Scans, multiple two dimensional cross-section images are taken and used to construct a three dimensional image of scanned objects. This allows the user to gain a sense of depth of the objects within the bag. It is one of the top technologies out there, but unfortunately it is very costly at about $\$ 500,000$ dollars and requires long amounts of time to construct such useful images.

\footnotetext{
${ }^{2}$ Photo furnished by InVision Technologies
} 
Lastly, backscatter imaging is another commonly used method for the detection of bulk level explosives (Figure 2.8). In this method, contrast within images allows the user to determine what types of objects are within the luggage.
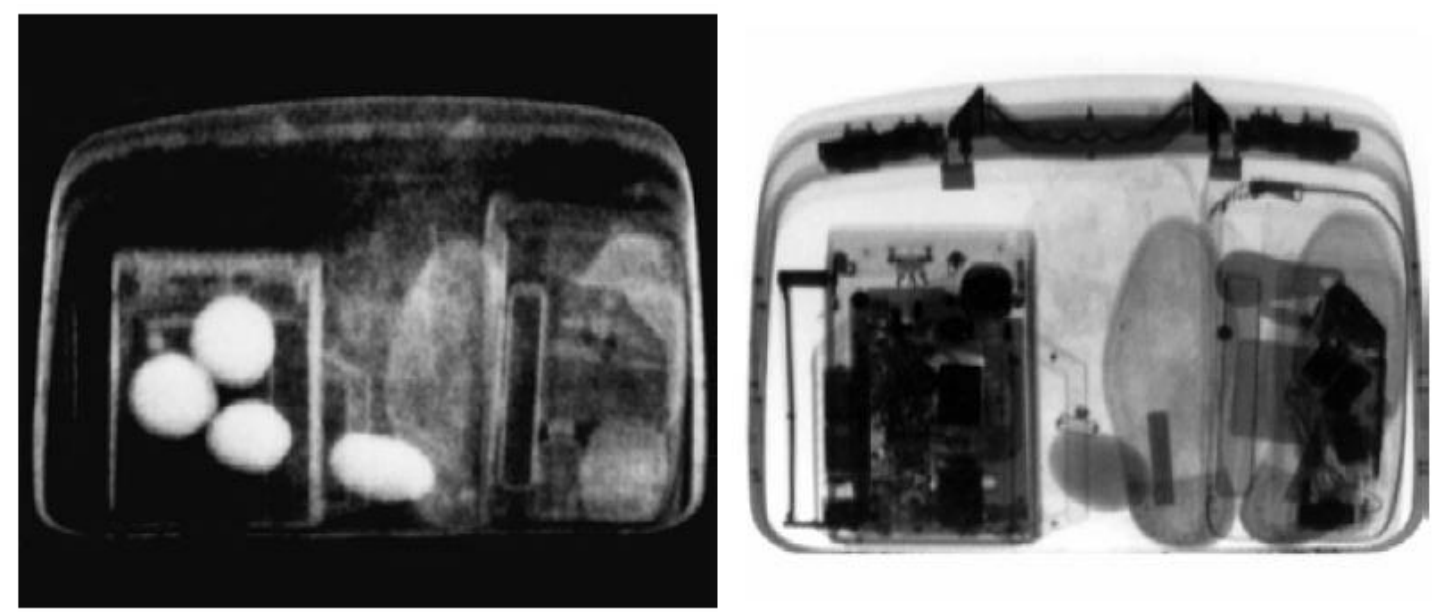

Figure 2.8 Backscatter image (left) of a suitcase with four potential threats identified while hidden within a radio which was not identified by the transmission $\mathrm{x}$-rays (right) ${ }^{3}$

Backscatter technology relies on differences in atomic numbers in order to identify potential threats. Those chemicals with high atomic numbers will appear brighter in the image, while those with lower atomic numbers will appear darker. This theory can lead to certain images being masked by high atomic number materials and thus multiple scans would need to be done in order to know what was concealed.

\subsubsection{Trace Detection Technology}

While there are some highly advanced technologies for bulk explosive detection out there on the market, there are other technologies out there to scan for those terrorists that have created and hidden IEDs without carrying them. In this case, one would look for any remnants of the explosive such as small

\footnotetext{
${ }^{3}$ Photo furnished by AS\&E
} 
particulates or vapor phase molecules. This type of detection is referred to as a trace detection system, and is the basis for gas sensor technology at the University of Rhode Island.

Let's first explore the various types of particulate detection techniques. Again, particulate detection refers to microscopic particles of the solid explosive material which adhered to either the surface of the user's hands or clothing when constructing and coming into contact with an IED. As a result of easy contamination when developing IEDs, particulate detection techniques such as GC/MS, chemical reagent based, and most notably ion-mobility spectrometry (IMS) are used for monitoring high traffic areas such as jetways.

Arguably, the most accurate particulate detection system is that provided by GC/MS (figure 2.9). This method requires the sample to be placed into a GC (gas chromatography) instrument for initial analysis.

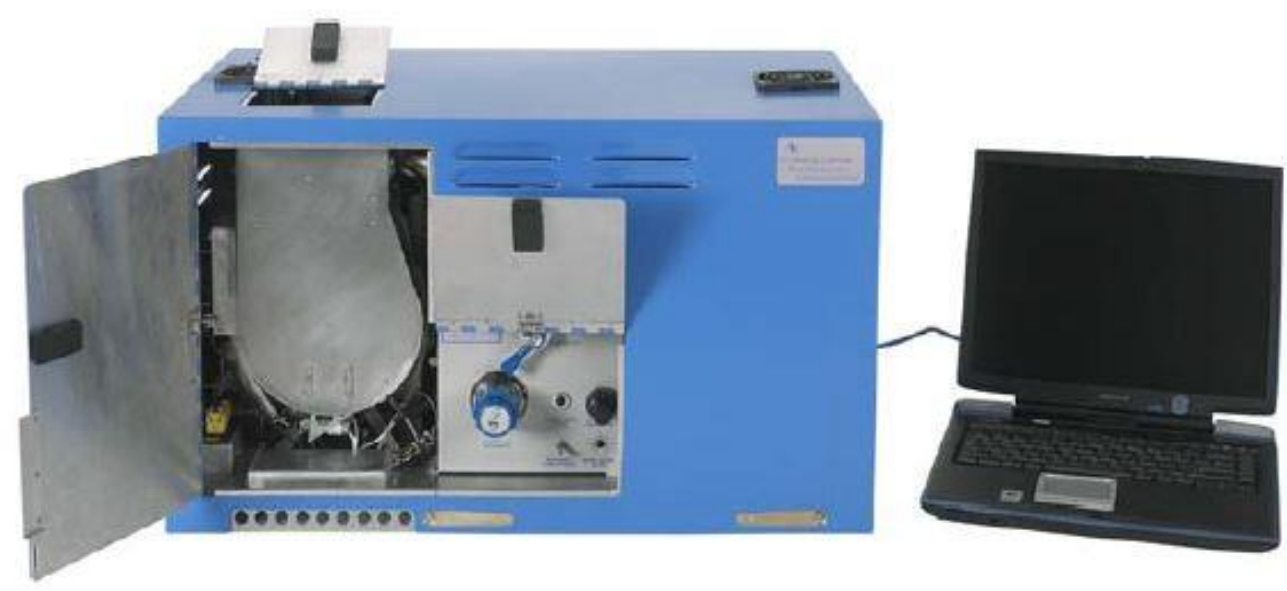

Figure 2.9 Portable GC/MS detection system used for particulate detection ${ }^{4}$

The sample is carried through the packed column using a carrier gas at temperature. The sample is separated into its individual components based on its

\footnotetext{
${ }^{4}$ Photos furnished by: Constellation Technology Corporation
} 
interaction within the column. The time that it takes for each individual component to move through the column and reach the detector is known as the retention time. These retention times are seen on a chromatograph where they can be compared with a standard explosive in order to determine whether or not there is an explosive present in the sample. In the event that the retention time of an explosive overlaps with a neighboring peak, MS (mass spectrometry) can be used to confirm the results. In MS, the chemical compound is ionized into small fragments. Based on these fragments and mass to charge ratios, the user can determine the atomic mass of the chemical in question. Although GC/MS is an effective method, such portable systems are costly and detection times can be lengthy, which hinders its ability for real-time monitoring.

On a relatively less expensive scale, chemical reagent based detection methods are also employed for particulate detection. In chemical reagent based methods, the user swipes a person's clothing or luggage, and applies a solution to the sample cloth. A positive response relies on a color change between the solution and the chemical in the sample. Although this method is much less expensive to implement compared to GC/MS, there are obvious drawbacks that include color discrepancy. It is solely based on the user's perception of color to determine whether or not an explosive is present. This being said, there can be user variation from time to time. In attempting to stop terrorist attacks, one would hope that a positive result would be better defined. 
An additional particulate detection method, which is probably the most widely used detection method today, is IMS (figure 2.10). Because of its quick response times, it can be found in nearly every airport across the United States.

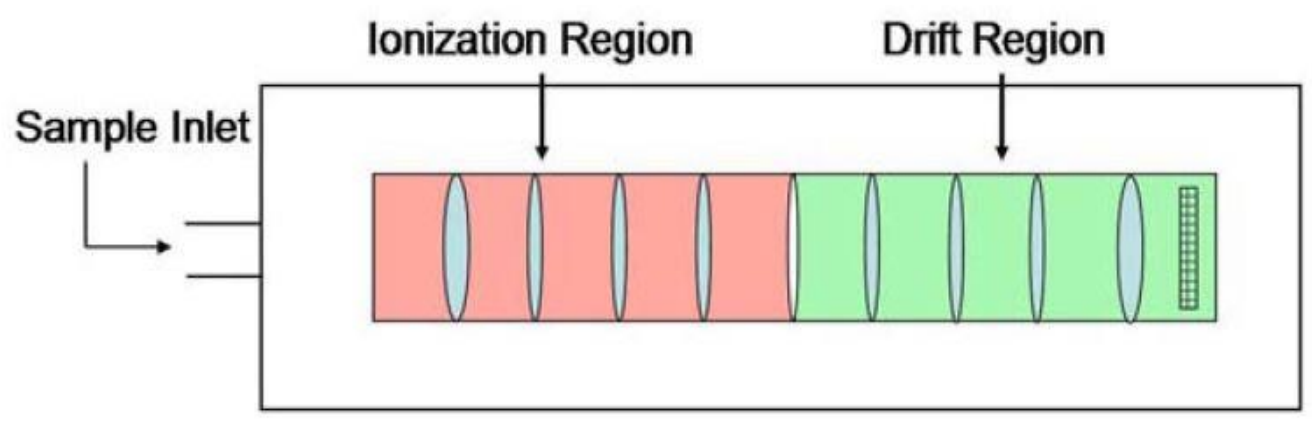

Figure 2.10 Components of IMS particulate detection system ${ }^{5}$

After the sample is collected by swiping a person's clothing or luggage, it is placed into the inlet of the IMS where the sample becomes ionized. The ionized particles then move through the drift region before reaching the detector. The drift times, which are the times it takes each ionized particle to reach the detector, are recorded as peaks on a chromatogram, similar to that of GC. These drift peaks are unique for each chemical and will ultimately be used to determine whether an energetic material is present. While detection times are quick, the sampling process becomes invasive by swiping an individual's clothing. Not only that, but similar to GC, there may be instances where it is tough to differentiate between peaks on the chromatogram.

If we recall the two types of trace detection, one is particulate detection, while the other is vapor detection. Vapor detection is the system that the group at

\footnotetext{
${ }^{5}$ Photo credit: Department of Justice Report
} 
the University of Rhode Island has developed. This type of detection relies solely on the vapor pressure of the explosives (figure 2.11).

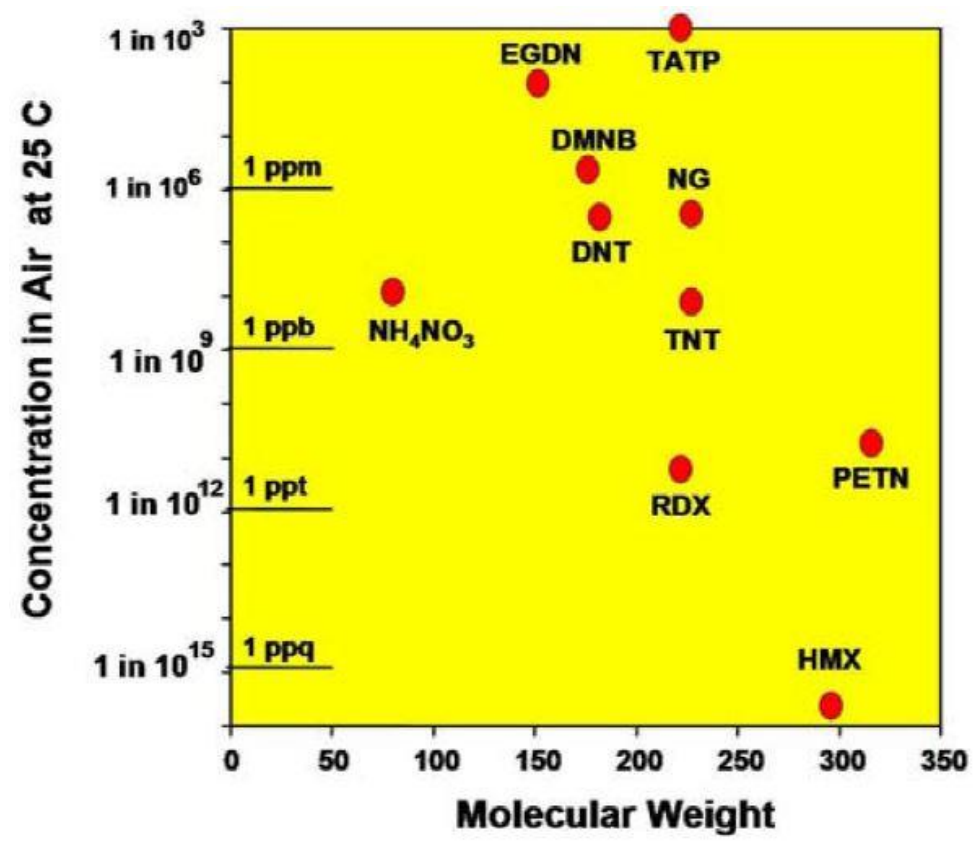

Figure 2.11 Concentration of some common explosives in air at room temperature ${ }^{6}$

At a given temperature, each of these chemicals vaporizes which creates a mixture of air and explosive. The concentration of explosive material in the air is a direct function of its vapor pressure [4]. For the purposes of our research, we explored an array of energetic materials spanning different vapor pressures. In comparison to other explosives, TATP has a relatively high vapor pressure, DNT has a lower vapor pressure and ammonium nitrate has the lowest vapor pressure of the three. The higher the vapor pressure, the more readily the chemical evaporates and concentrates the nearby air [4].

As far as vapor phase detection goes, the gold standard is still the use of canines. With a minimum detection level in the parts per trillion, drug sniffing canines are used in airports across the country. They not only have the ability to

\footnotetext{
${ }^{6}$ Photo credit: Department of Justice Report
} 
monitor the air, but they can pin point the region where the explosives were kept with great accuracy. Even though drug sniffing dogs have the lowest detection limit for these energetic materials, they pose some major problems that need to be addressed. The first issue is cost. To train such dogs to become accurate enough to use for airport monitoring, requires an immense of amount of time and money. Including the costs of health insurance, and food the total estimate to train one of these dogs is upwards of $\$ 100,000$. This being said, the return on these dogs is even less cost effective because their keen sense of smell only lasts a number of years. After a few years, a new dog must be trained in order to replace the old dog. Although they do have the ability to measure at the parts per trillion level, they can only do this for a number of hours before they need a break and thus makes them a non-continuous detection method. With such immense gaps in the top detection systems, a new method is warranted that can continuously monitor the air at a relatively inexpensive rate, which is the goal of the research at the University of Rhode Island.

\subsubsection{Dr. Otto Gregory SSTP Laboratory Group Accomplishments}

Up to this point, great strides in trace explosive detection have been made by Professor Gregory's group as part of the center for Sensors and Surface Technology Partnership (SSTP) at the University of Rhode Island. Multiple sensing platforms (conductometric and thermodynamic) have been developed to integrate the metal oxide catalyst for analytes of interest at various concentrations (figure 2.12). With a focus on vapor phase detection, a sensor was developed that will continually "sniff" the air, similar to that of a dog, which has the ability to 
closely monitor jetways, train stations, and other high traffic areas for potential threats posed by terrorists [5].

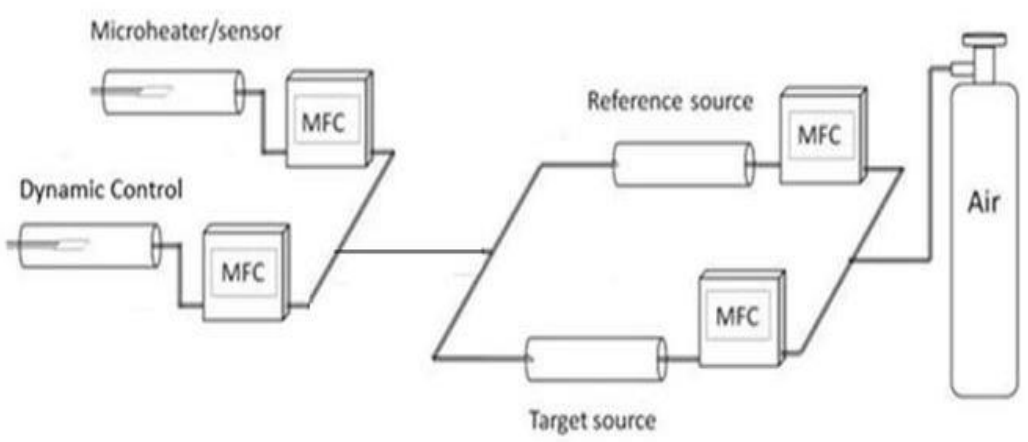

Figure 2.12 Schematic of the trace detection system employing dynamic control.

The sensing apparatus shown above, employs a "dynamic" testing protocol whereby one of the gas sensors is fabricated with a catalyst while a second microheater is used as the "dynamic" control for the purpose of testing. The dynamic control takes into account the sensible heat effects due to humidity, particles in the air, etc. By subtracting out signals obtained from the dynamic control, we can see only those affects due to the analyte of interest (figure 2.13). 


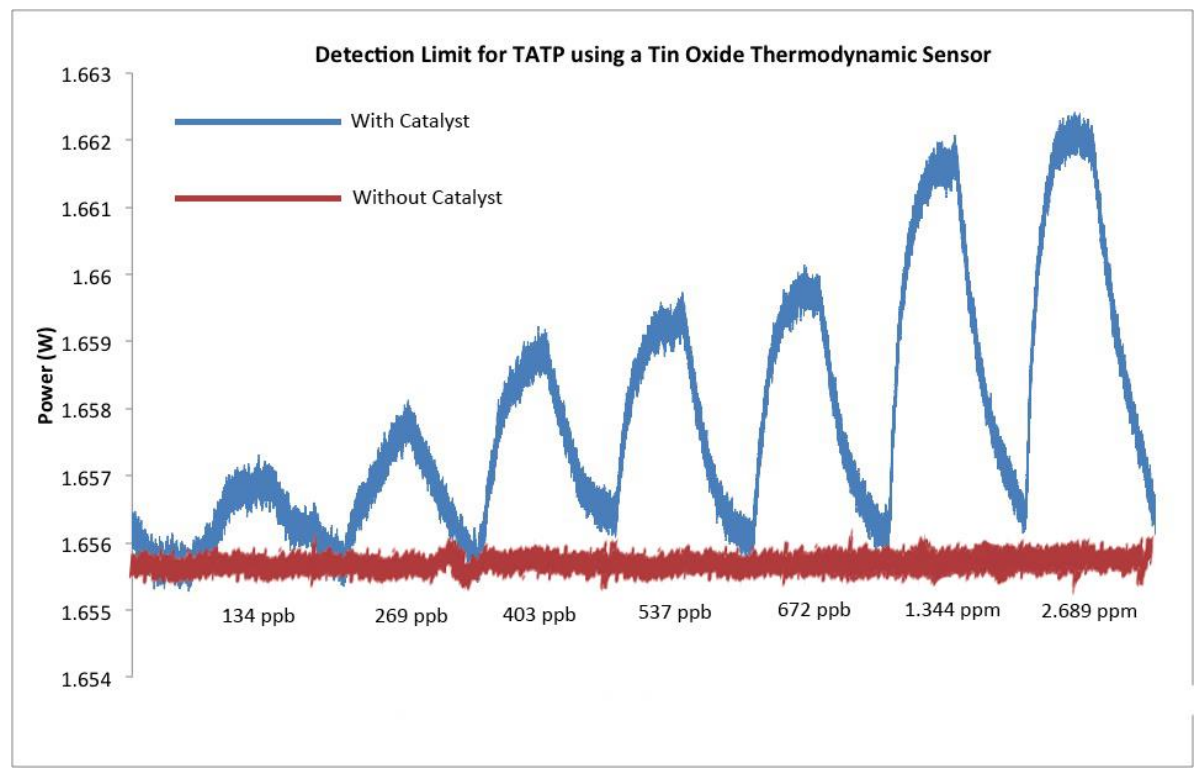

Figure 2.13 Thermodynamic sensor response to TATP from both the catalyst coated sensor (blue) and the dynamic control (red)

The thermodynamic approach monitors the power changes associated with the decomposition of vapor phase molecules as they adsorb to the surface of the catalyst and rapidly decompose. Catalytic decomposition of such energetic chemicals results in a heat effect that can be measured by our thermodynamic sensor. It is certainly important to maintain a reference temperature when using this approach, or any other approach for that matter, considering the various environmental conditions in which the sensor operates. Again, by subtracting out the power change resulting from the dynamic control, the power change due to the decomposition of the analyte is measured.

An additional sensing platform developed at the University of Rhode Island is referred to as a conductometric sensor. In this platform, electrical changes in the resistance of the catalyst are measured as a result of analytes interacting with the catalyst (figure 2.14). 


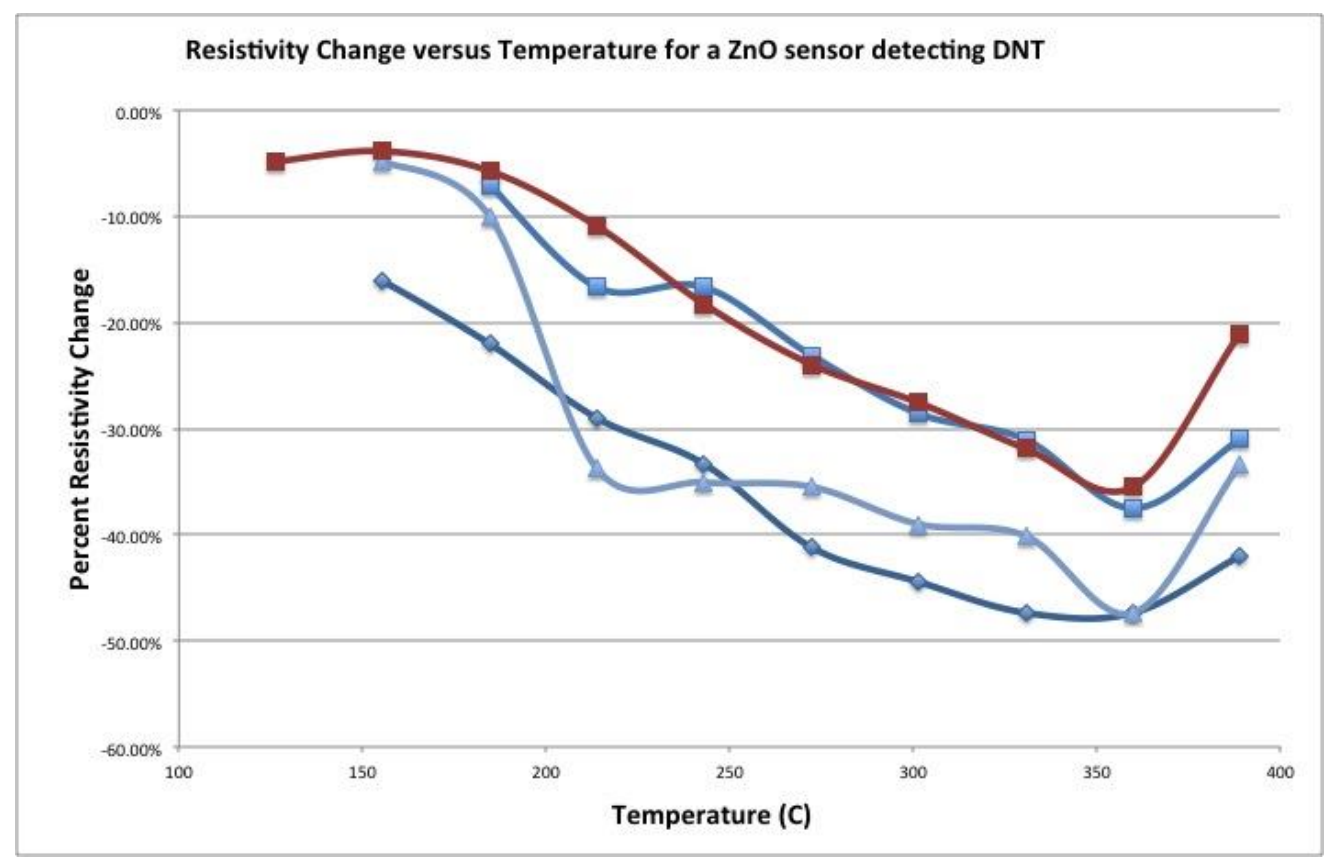

Figure 2.14 Conductometric response to 2, 6-DNT employing a $\mathrm{ZnO}$ catalyst at various temperatures

The conductivity of the catalyst changes with temperature and an optimal response is observed at a specific temperature, providing a redundant measurement that is complimentary to the heat effect measured. In conjunction, both the conductometric sensor and the thermodynamic sensor can be fabricated using the same catalyst and thus provides orthogonality to the measurement. By acquiring two independent signals from a single exposure, a unique signature for each explosive compound will be realized. It will enable us to determine if there is a potential threat, what type of threat and at what concentration the threat is present.

\subsubsection{Thermodynamic Sensing Improvements}

Canines have the ability to detect energetic materials at the parts per trillion level, and are the gold standard in detection. Thus we continue to move towards lowering detection limits that could compete with a dog's nose. The 
minimum detection limit is the concentration of analyte measured as a sensor response relative to the noise flow. One approach used to improve the thermodynamic sensor is the use of pre-concentration (figure 2.15).

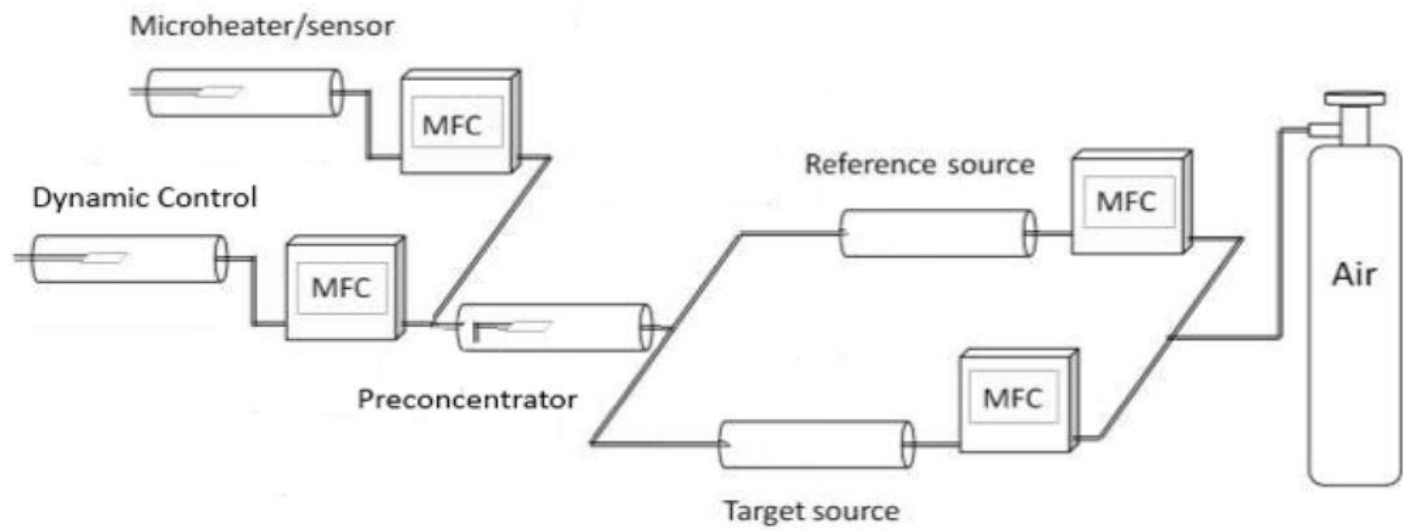

Figure 2.15 Schematic of pre-concentration test bed for thermodynamic sensing applications The test bed is the same as the dynamic approach with the exception of a preconcentrator microheater placed upstream from the gas sensor and dynamic control. The pre-concentrator (heater) is coated with a polymer that is tailored to concentrate vapor phase explosive molecules. After certain adsorption time, the pre-concentrator is heated, allowing for desorption of these explosive molecules. This mechanism allows for a highly concentrated burst of analyte to be delivered to the sensors all at once, thus increasing the signal for a given vapor phase concentration. It is imperative to the design to fabricate the pre-concentrator with a polymer that possess both adsorption and desorption properties (table 2.1). 


\begin{tabular}{llll}
\hline Desorption Efficiency of TNT at $\mathbf{1 7 0 C}$ & $\begin{array}{l}\text { TotalAmount } \\
\text { Adsorbed }\end{array}$ & $\begin{array}{l}\text { TotalAmount } \\
\text { Desorbed }\end{array}$ & \% Desorbed \\
\hline Poly(ethylene terepthalate) & 2.40 & 0.11 & $5 \%$ \\
\hline Tenax & 7.88 & 0.50 & $6 \%$ \\
\hline Poly(2,6-dimethyl-1,4-phenyline oxide) & 6.60 & 0.46 & $7 \%$ \\
Poly(4-vinyl phenol) & 10 & 0.80 & $8 \%$ \\
\hline Polystyrene & 6.22 & 3.61 & $58 \%$ \\
\hline Teflon & 2.32 & 2.48 & $107 \%$ \\
\hline
\end{tabular}

Table 2.1 Properties of polymer candidates for pre-concentrator applications

Most polymers possess only one of these characteristics. Not only that, but they need to bind to our target analytes and so specificity becomes key in choosing a correct polymer. Looking at table 2.1, at times there is a major discrepancy between the amounts of analyte adsorbed and desorbed. For our purposes, polystyrene was selected as the ideal candidate for pre-concentrator applications and was doctor-bladed onto the additional microheater upstream as shown in figure 2.15. Polystyrene not only showed specificity towards nitrogen based explosives, but the quantity that was adsorbed on the surface was promising as well. When detecting such low levels of energetic materials, it was very important that the sensor collect as many molecules as possible and so polystyrene was down-selected as the polymer of choice. A number of tests were completed using the pre-concentrator mechanism with conclusive results (figure 2.16). 


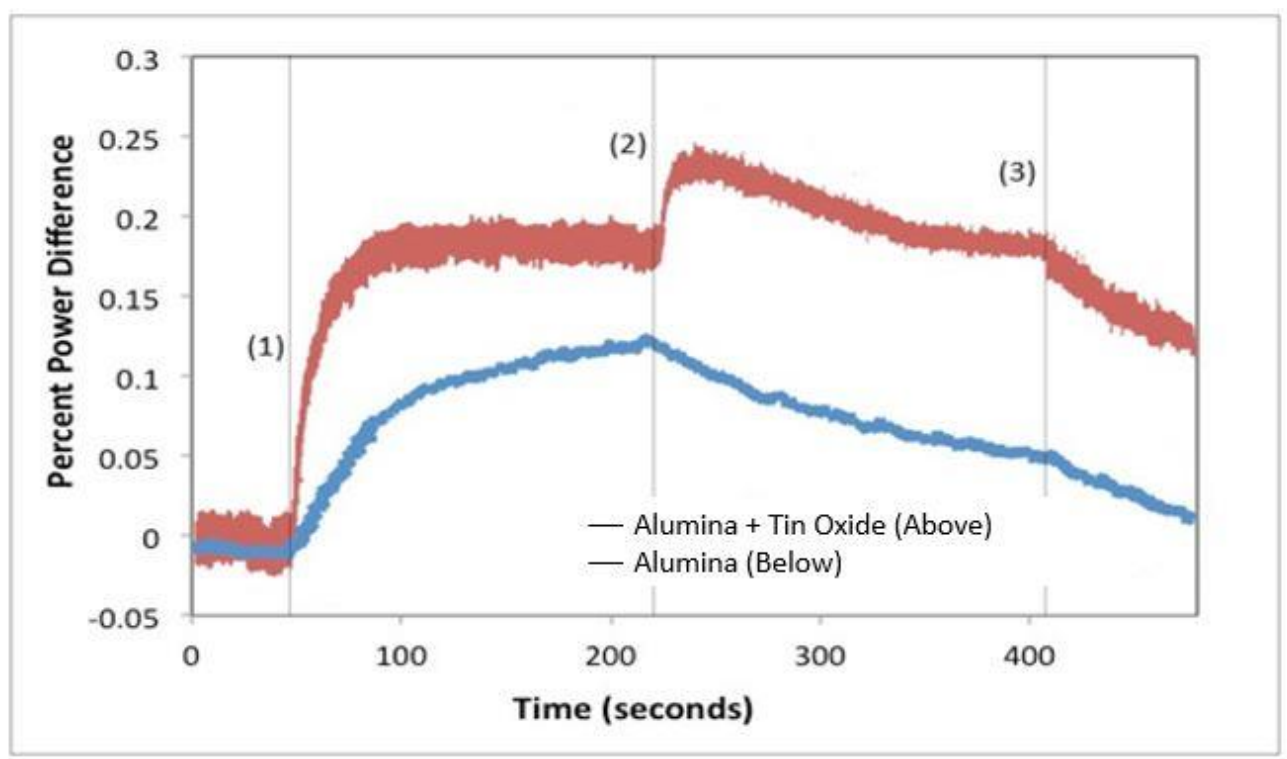

Figure 2.16 Preconcentrator duty cycle beginning with the introduction of 2, 6-DNT vapor to the preconcetrator (1), followed by preconcentrator thermal desorption of DNT (2), and lastly the preconcentrator is turned off while reference gas is reintroduced to the system (3)

The detection improvement when using pre-concentration can easily be identified when comparing sensor responses. In figure 2.17, a peak in the data can be seen at step two after thermal desorption of 2, 6-DNT. This peak represents the response due to the concentrated burst of analyte that is sent directly to the sensor for detection purposes. Based on the power differences observed in the data, there was nearly a 50\% increase in signal response when employing the preconcentrator upstream and thus further improved minimum detection limits.

The most recent improvement in the detection system and the focus of this research has been the fabrication of metal oxide nanowires. Since a MEMS based device (figure 2.17) has been in the developmental stages for quite some time now, it is necessary to develop a sensor design that increases the surface area without adding area to the sensor footprint [6]. 


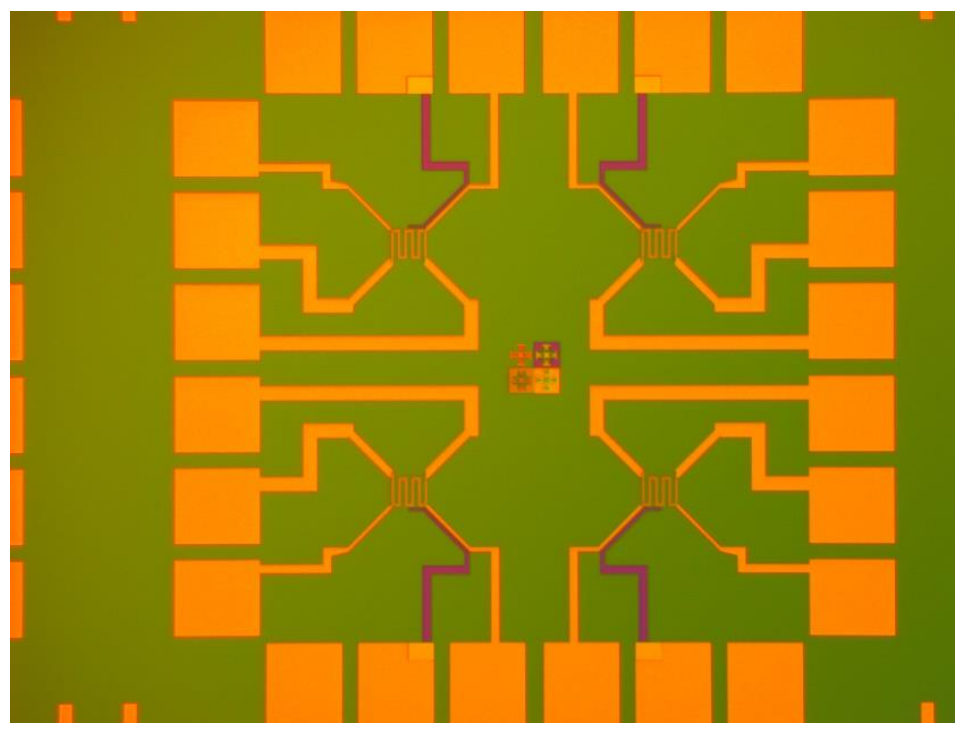

Figure 2.17 Schematic diagram of the MEMS based platform for chemical sensing The MEMS based device is not only smaller in size than the current solid state device, but it is also fabricated with 4 sensors to form a sensing platform with built in redundancy. With such a reduction in size, it is necessary to increase the surface area of the catalyst by orders of magnitude to make the transition.

A number of metal oxide nanowires have been investigated for chemical sensing purposes. Specifically, zinc oxide, copper oxide and iron oxide have been directly fabricated on our solid state thermodynamic sensor and have been tuned for targeting threat molecules. They have been fabricated using a combination of sputter deposition techniques followed by hydrothermal reactions or direct oxidation, and then characterized by SEM, XRD and XPS. Ultimately, they will enable lower detection limits and greater specificity towards potential threats. 


\section{REFERENCES}

[1] Miller J. S., Johansen R. T. Fracturing Oil Shale with Explosives for In Situ Recovery. American Chemical Society 151 Retrieved (2015)

[2] Agrawal J. P., Hodgson R. D., Organic Chemistry of Explosives. John Wiley \& Sons (2007)

[3] Ewing R. G., Atkinson D. A., Clowers B. H., Direct Real-Time Detection of

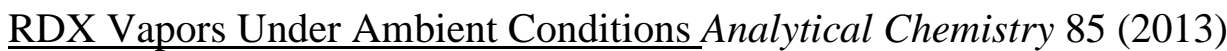

[4] Arshak K., Moore E., Lyons G. M., Harris J. and Clifford S. $\underline{\text { A Review of Gas }}$ Sensors Employed in Electronic Nose Applications. Sensor Review vol. 24 (2004): 181-198

[5] Gazit I. and Terkel J. Explosives detection by sniffer dogs following strenuous physical activity. Applied Animal Behaviour Science 81.2 (2003): 149161.

[6] Camara M., James F., Breuil P., Pijolat C., Briand D., de Rooij N. F., MEMS$\underline{\text { based porous silicon preconcentrators filled with Carbopack-B for }}$ explosives detection. Procedia Engineering vol. 87 (2014): 84-87. 


\section{CHAPTER 3}

\section{METHODOLOGY}

\subsection{Thermodynamic Gas Sensor}

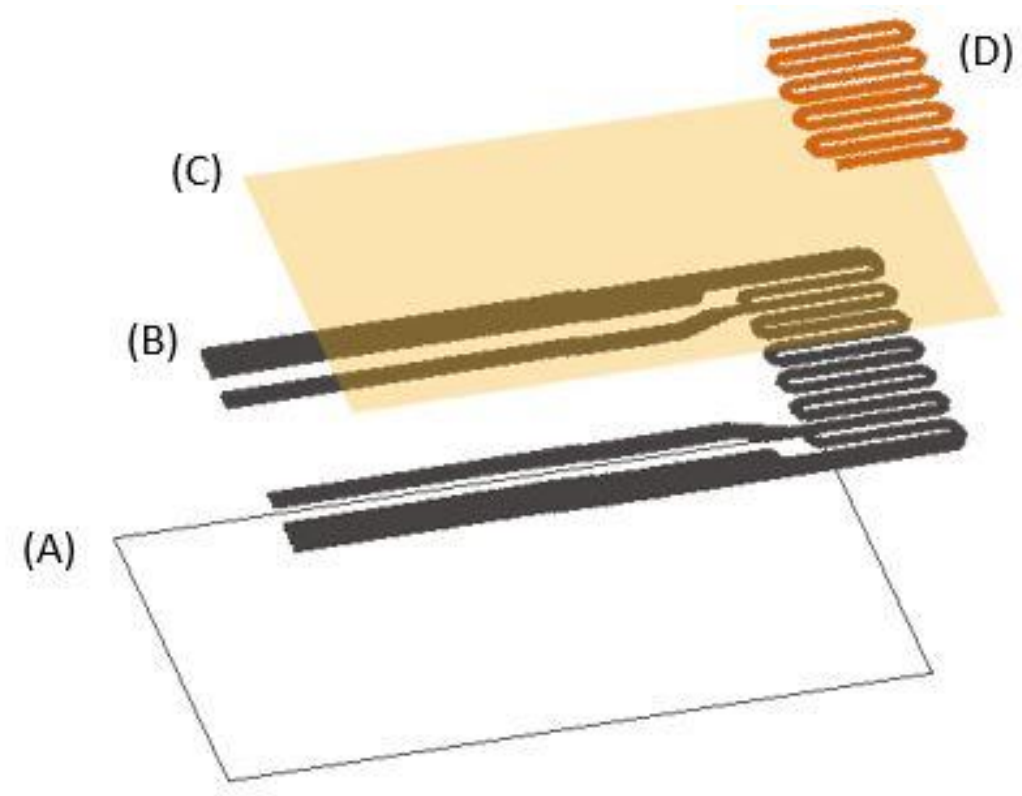

Figure 3.1 Schematic of the various layers comprising the thermodynamic sensor. The alumina ceramic substrate (A), Ni microheater (B), aluminum oxide passivation layer (C) and the metal oxide catalyst (D)

The solid state sensor developed at the University of Rhode Island is comprised of multiple layers of thin film materials as shown in figure 3.1. The substrate of the sensor is a lapped alumina (99.6\% purity) manufactured by PM Industries. It is pre-scribed on either side so that each sensor can be separated with ease. The scribe also ensures correct alignment for masking steps in photolithography. The alumina substrate was chosen because of its ability to withstand the working 
temperatures necessary for the catalytic decomposition of the energetic materials without cracking.

\subsubsection{Nickel Microheater Patterning}

To create the Ni microheaters used for the dynamic control approach, a number of lithography steps are repeated to form the final device. The alumina substrate is RCA cleaned; i.e. the substrate is cleaned with acetone (99\% pure), then methanol (99\%) and lastly deionized water to rid the surface of any particulates. A negative photoresist solid film is then applied to the surface of the alumina substrate and rolled out so that it is rid of all air pockets. The substrates are then placed on a hot plate at 90C for ten minutes, also known as "pre-baking" to ensure that the resist stays in place during UV exposure. The alumina plates are then placed on the UV exposure stage where they are aligned with the mask containing the pattern for the Ni microheater. The plates are then exposed to UV radiation for a period of seven seconds so that the mask pattern is transferred to the substrates. The plates are then developed in a solution of $300 \mathrm{mLs}$ deionized water, $5 \mathrm{mLs}$ developer solution and $0.5 \mathrm{~g}$ sodium bicarbonate, which activates the developer solution. The ceramic plates are developed for 40 seconds to open windows within the resist for sputter deposition of Ni. When using negative resist, the resist that is exposed to UV radiation becomes cross-linked, non-polar, and thus does not dissolve in the polar developer solution. The excess resist is rinsed off again with deionized water before being exposed for an additional 20 seconds to ensure a clean pattern for sputter deposition. A final post bake step for 20 minutes at $100 \mathrm{C}$ is then completed before the $\mathrm{Ni}$ is applied. 
The sensors are transferred to the sputtering machine MRC 822 where at 200W power and 9mTorr Argon gas (99.999\% purity) $4.5 \mu \mathrm{m}$ of $\mathrm{Ni}$ is deposited onto the sensors [1]. The sensors are then returned to the Photolithography Laboratory where liftoff is completed. In this process, underlying photoresist is removed by agitating the substrates in high purity acetone. After liftoff is complete, the Ni microheater shown in figure 3.1 remains. The microheaters are then nitrogen annealed using industrial grade nitrogen gas, in a Mellen tube furnace at 900C for a period of five hours. This step ensures the best electrical properties for the microheater by aligning $\mathrm{Ni}$ atoms and creating a uniformity across the different sensors [1]. When fabricating the sensors it should be obvious that the goal is to replicate them as best as possible including their electrical properties. The only variables that should remain when testing multiple sensors is analyte concentration, which is why the annealing step is necessary. It also allows the sensors to be more durable to the working temperatures since they are initially exposed to temperatures well above what is required, during the annealing process.

\subsubsection{Passivation Layer Fabrication}

The next step in the fabrication of the gas sensors, is the application of the aluminum oxide passivation layer. This layer is created by first placing the sensors in the sputtering machine MRC 8671 and sputter depositing $1 \mu \mathrm{m}$ of aluminum oxide in 9mTorr Argon conditions [1]. Sputter deposition is completed in two hour increments to prevent pin holes from forming in the film, which could eventually lead to a short in the electrical components [1]. In addition to a 
sputtered film, a porous, aluminum oxide cement is doctor-bladed over the serpentine of the microheater. The aluminum oxide cement has multiple merits for our sensor in that it helps to protect the underlying Ni microheater while also providing improved responses to explosive analytes (figure 3.2).

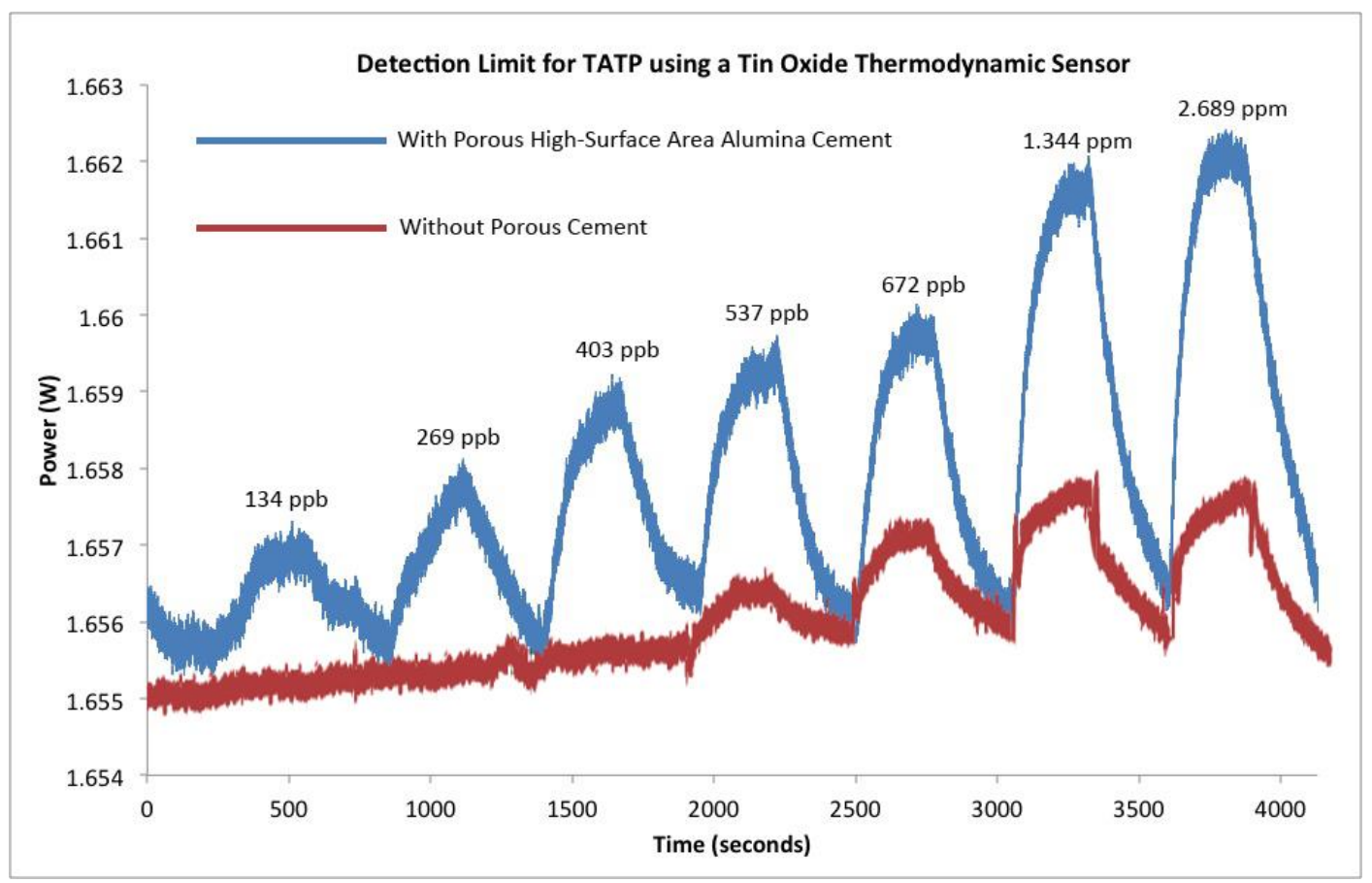

Figure 3.2 Sensor responses to various concentrations of TATP showing the merit of using a porous alumina cement (blue) versus no cement (red)

The graph shown in figure 3.2 depicts a concentration test, where the sensors are held at a constant temperature while being exposed to various concentrations. The amplified signals, which were shown in blue, were the result of the improved sensor fabrication technique by adding a porous alumina cement over the serpentine of the Ni microheater. The alumina cement adds additional surface area to the sensor which allows for greater interaction between explosive vapor molecules and the catalyst surface and thus eliciting a larger response.

\subsubsection{Metal Oxide Nanowire Catalysts}


After forming a porous alumina layer on the sensor, the catalyst was deposited on the microheater surface. This step is crucial in that the catalyst decomposes the energetic material and thus provides a sensor response. Catalysts are chosen based on properties such as melting point and oxidation states. Since these catalysts will be running at temperatures in the range of 300-400C they obviously should have melting temperatures above this range. Catalysts are also selected based on the number of oxidation states. Multiple oxidation states allow for a transfer of electrons to occur and ultimately the interactions that are desired. Originally, sputter deposited catalysts were used as the basis for the metal oxide catalyst, but the most recent improvements have been related to the development of metal oxide nanowires as catalysts.

Zinc oxide nanowires were grown by the hydrothermal reaction of zinc metal with ethylene diamine in a multi-step process. High purity zinc $(99.9 \%)$ is rinsed with acetone, methanol and water to ensure a pristine surface [2]. The zinc surface was placed in a teflon crucible and submerged in a $3.5 \mathrm{M}$ solution of ethylene diamine and water [2]. The crucible was placed in an autoclave for 10 hours at 140C to induce the growth of the zinc oxide nanowires [2]. The reaction is limited to the amount of zinc present on the surface and so once the foil is covered with nanowires, the reactions seizes to proceed. After the hydrothermal reaction (eq. 1-3) is completed, the zinc oxide nanowires are then deposited onto the sensor for use [2]. 


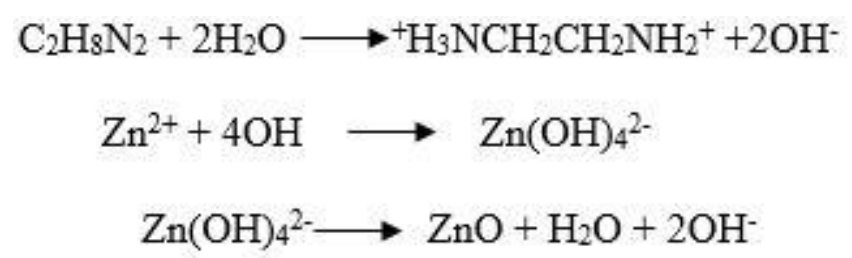

Due to the variety of metal oxide candidates, it was natural to explore methods in which thin films could be deposited directly on the sensor surface for the purpose of nanowire growth. This being said, copper oxide nanowires were explored for their ease in fabrication. A fine $3 \mu \mathrm{m}$ copper film was deposited on top of the porous alumina cement using a MRC $8667 \mathrm{RF}$ sputtering machine [1]. The sputter conditions included the use of ultra-high purity argon (99.999\%) at a chamber pressure of $9 \mathrm{mT}$ Torr. This pressure not only ensures an ideal plasma is achieved, but also so that the mean free path for the copper atoms is larger and allows them to reach the surface of the work piece. Once the film has been deposited onto the sensor surface, the sensors remain in the sputtering machine for 24 hours to ensure a dense film. This allows the film to rid itself of any argon trapped inside resulting from the sputtering process. The sensors are then removed from the sputtering machine and placed inside the Mellen tube furnace for nanowire growth. They are annealed in stagnant air at $400 \mathrm{C}$ for a period of five hours [3]. The sensors can then be used for testing applications.

Additionally, iron possess multiple oxidation states and so iron oxide nanowires were considered as an attractive candidate for a catalyst. Not only that, but they can be developed easily on the surface of the sensors in a similar mechanism to that of the copper oxide nanowires [4]. Again using the MRC 8667 RF sputtering machine, a fine $3 \mu \mathrm{m}$ iron film was developed on the sensor surface 
in 9mTorr Argon pressure [1]. The samples were allowed to sit in the sputtering machine overnight and then transported to the Mellen furnace. The parameters for iron oxide nanowires is 400C for one hour of time [4]. These parameters create a high-density, nanowire film which is ideal for sensing applications as both a catalyst and catalyst support.

\subsection{Dynamic Sensor Testing}

Similar to the approach used for pre-concentration, the following test setup was used to isolate the effects of a metal oxide nanowire catalyst (figure 3.3).

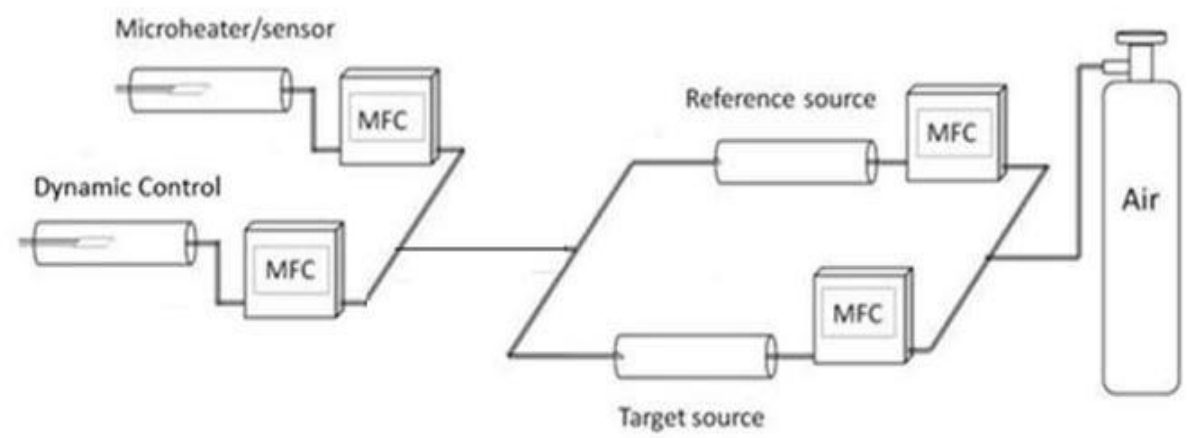

Figure 3.3 Schematic of electronic detection system using dynamic control in conjunction with nanowire catalyst.

The dynamic protocol utilizes a carrier gas, four Alicat mass flow controllers, stainless steel piping, two chambers for sample prep, and two sensor holders. The desired explosive, in this case either TATP, 2, 6-DNT or ammonium nitrate, is loaded into the "Target" stainless steel chamber while the "Reference chamber" is left empty. Compressed air is used as a carrier gas to deliver various concentrations of explosive to the sensors downstream. The two mass flow controllers upstream are used to vary the concentration of explosive to the sensors. The concentration is varied by flowing some compressed air through the 
"Target chamber" while the rest through the "Reference chamber". Depending on the explosive used and its vapor pressure, a certain amount of explosive is vaporized and carried by the flowing gas to the active sensor array. This explosive-rich vapor is then mixed with air from the "Reference source" to create the desired concentration. The mass flow controllers deliver equal parts of the explosive-air mixture to both the sensor and dynamic control. As stated previously, the dynamic control possesses no catalyst and is used to monitor sensible heat effects.

\subsubsection{Testing Techniques using the Dynamic Approach}

The thermodynamic detection method relies on the catalytic decomposition of energetic materials as they adhere to the sensor surface [5]. This type of reaction is completed at an optimal temperature which is the working temperature of the sensors and is maintained throughout any testing. In order to measure the decomposition response, the amount of power required to maintain the sensor temperature is monitored using a four point probe method [5]. As the analyte decomposes, it releases an amount of energy, in the form of heat, which can be monitored by the change in the electrical power of the sensor to maintain temperature. Thus, a power change in the sensor at a given temperature is used to determine whether or not an explosive vapor molecule is present.

There are numerous methods used to test the ability of the sensors. These methods include a SCAR test, a Single-Step test and lastly a Concentration test. When exploring new sensors or catalysts, it is important to find the temperature which elicits the greatest response towards analytes. This type of testing is known 
as a SCAR test. During a SCAR test, the temperature is increased in intervals while being exposed to a single concentration of analyte. This allows the user to see the temperature that produces the greatest signal response to a given analyte. Once the response is optimized, we address various concentrations of explosives. The Single-Step test is used first for a general comparison of signals. It is the quickest test in that it only requires 15 minutes of response time and ultimately provides insight as to whether or not the sensor is responding to the analyte. The test consists of three intervals. During the first interval, compressed air is delivered to the sensors through the "Reference chamber". This provides a baseline signal. After five minutes, the analyte is delivered to the sensors through the "Target chamber" at 100 SCCM (standard cubic centimeters). This carries explosive-rich air to the sensors to test for a response. Since decomposition of the explosive releases energy, that energy is monitored as a power change in terms of a sensor response. After five minutes of exposure, the air is reintroduced towards the "Reference chamber" and the sensor response returns to the baseline.

After screening the sensors for functionality, various concentrations of analyte were passed over the sensors to establish the minimum detection limit. Sensor temperatures were scanned to establish the optimal working temperature for the reaction to occur. The data is collected at five minute intervals during which the reference gas is delivered and then a new concentration of explosive vapor is introduced. The concentration of the energetic material had been previously calculated as a function of their vapor pressures, flow rates, and ambient conditions. The sensors are interfaced to a VM Heater data acquisition 
system that continuously logs 10 data points/sec [5]. After collection, the data is analyzed i.e. multiple response curves are generated that display the power changes associated with the sensors as a function of time. 


\section{REFERENCES}

[1] Hill R. J. Physical Vapor Deposition. Temescal (1986) ed. $2^{\text {nd }}$

[2] Yajun J. One-step method for growing of large scale $\mathrm{ZnO}$ nanowires on zinc foil. Materials Letters 138 (2015): 92-95.

[3] Chopra N., Shi W. and Lattner A. Fabrication and characterization of copper $\underline{\text { oxide }(\mathrm{CuO}) \text {-gold }(\mathrm{Au}) \text {-titania }(\mathrm{TiO} 2) \text { and copper oxide }(\mathrm{CuO}) \text {-gold }}$ (Au)-indium tin oxide (ITO) nanowire heterostructures. Materials Characterization 96 (2014): 71 -

77.

[4] Shen X., Song F., Yang X., Wang Z., Jing M., Wang Y., $\underline{\text { Hexaferrite/ } \alpha \text {-iron }}$ composite nanowires: Microstructure, exchange-coupling interaction and microwave absorption. Journal of Alloys and Compounds 621 (2015): $146-153$

[5] Mallin D. Increasing the Selectivity and Sensitivity of Gas Sensors for the Detection of Explosives. University of Rhode Island M.S. thesis (2014). 


\section{CHAPTER 4}

\section{RESULTS}

\subsection{Metal Oxide Nanowire Catalysts}

Earlier gas sensor research relied on sputtered catalysts to aid in the decomposition of the explosive molecules and although successful, would not enable the transition to a MEMS based platform. The transition towards a MEMS

device requires that all components of the solid state device be reduced in size and the need for a much greater surface area catalyst was apparent. The choice to fabricate metal oxide nanowires as the catalyst was necessary in order to create a viable signal while reducing the size of the sensor platform.

\subsubsection{Zinc Oxide Nanowires}

The hydrothermal reaction of zinc to form zinc oxide nanowires requires 10 hours inside an autoclave (figure 4.1) [1-2]. 


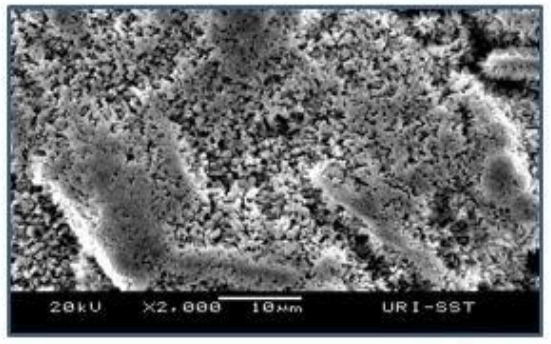

2 HOURS

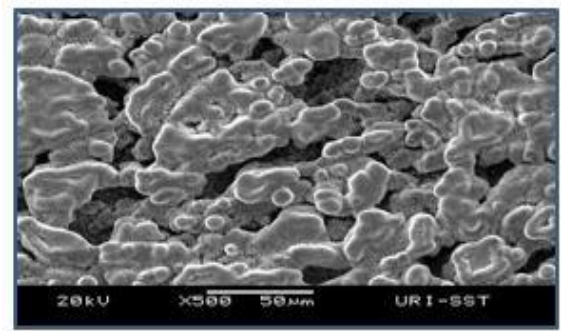

6 HOURS

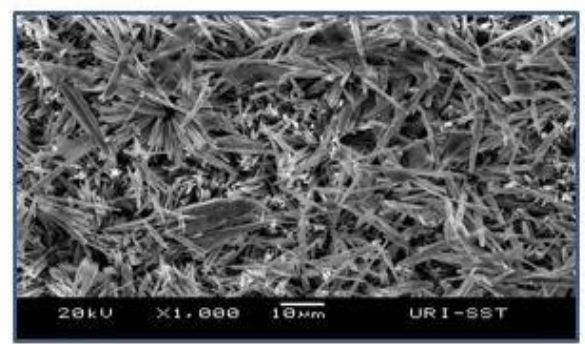

10 HOURS

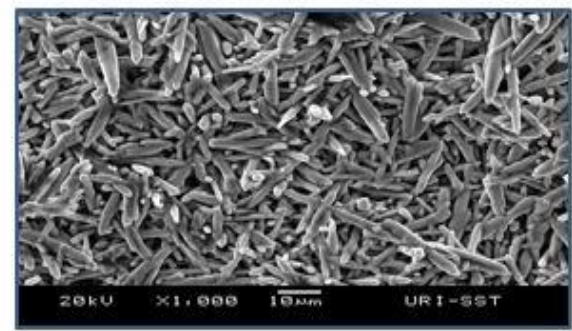

8 HOURS

Figure 4.1 SEM micrographs depicting the growth of zinc oxide nanowires

Over the first six hours of growth within the autoclave, small globules can be seen signifying a change in the surface chemistry of the foil. Upon eight hours of heating, small needle-like structures can be seen and finally, at ten hours, distinct nanowires can be observed. It is proposed that at the beginning of the hydrothermal process, the presence of the zinc ions with hydroxyl ions form small nanoparticles (as soon as the two hour mark in figure 4.1) [1-2]. As a result of the high pressures and gas-liquid equilibrium within the autoclave, these nanoparticles are forced to assemble in needle-like structures, forming the nanowires observed at the ten hour mark. Since there were no longer zinc ions available once the surface was covered with nanowires, then the reactions no longer proceed [1-2].

Metal oxide nanowires were a major improvement over the sputter deposited catalyst in terms of surface area available for analyte interaction (figure 4.2). While the transition to a MEMS based platform is important, an increase in 
surface area of the catalyst represents a major advance in both selectivity and sensitivity to the existing sensor platform.

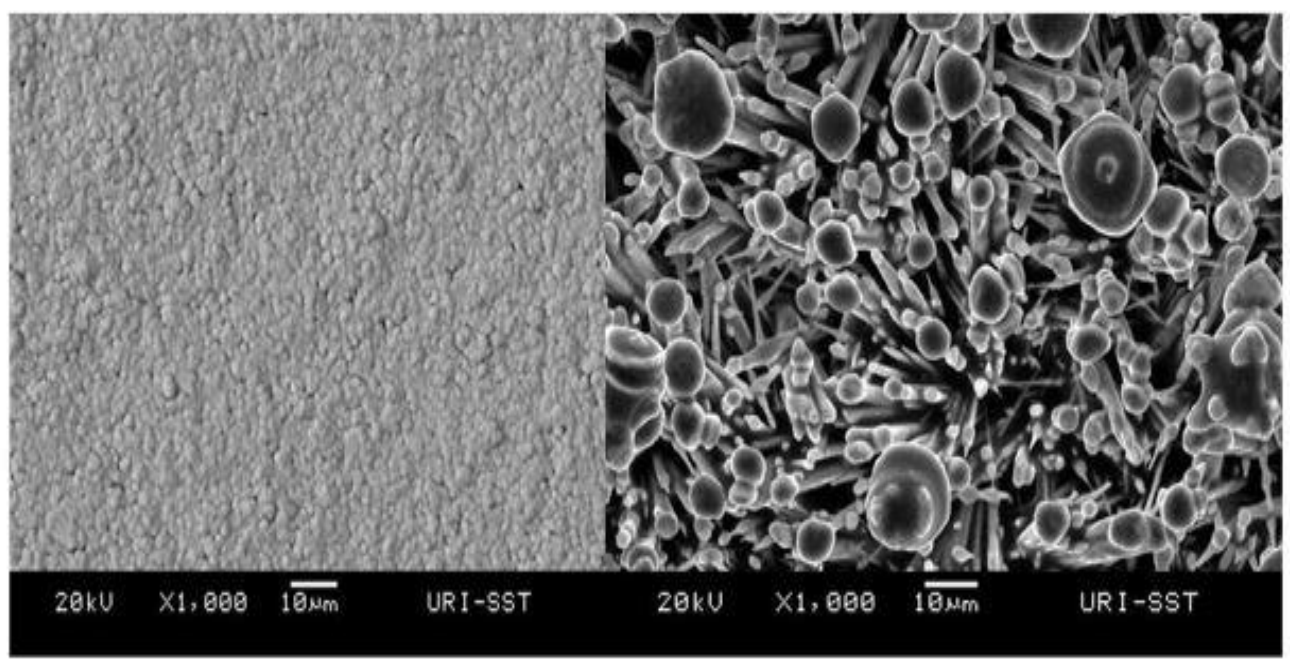

Figure 4.2 SEM micrographs comparing the sputter deposited zinc oxide film (left) to the high surface area zinc oxide nanowire catalyst (right).

Although a sputtered zinc oxide catalyst has shown merit in the past, the asdeposited film is somewhat featureless. However, the zinc oxide nanowires exhibit features not previously seen in the sputtered catalyst, which add considerable surface area. This nano-structure is useful for catalyst development in 2 ways; they can be used as either a stand-alone catalyst or as catalyst support for other metal oxide catalysts. Thus, the nanowires provide additional surface area for other sputtered deposited catalyst films so that multiple decomposition reactions may be optimized (figure 4.3). As a catalyst support, the nanowires are used solely for the purpose of increasing viable surface area of an additional catalyst whereas, as a stand-alone, the nanowires are the catalyst of choice for the decomposition reaction. 


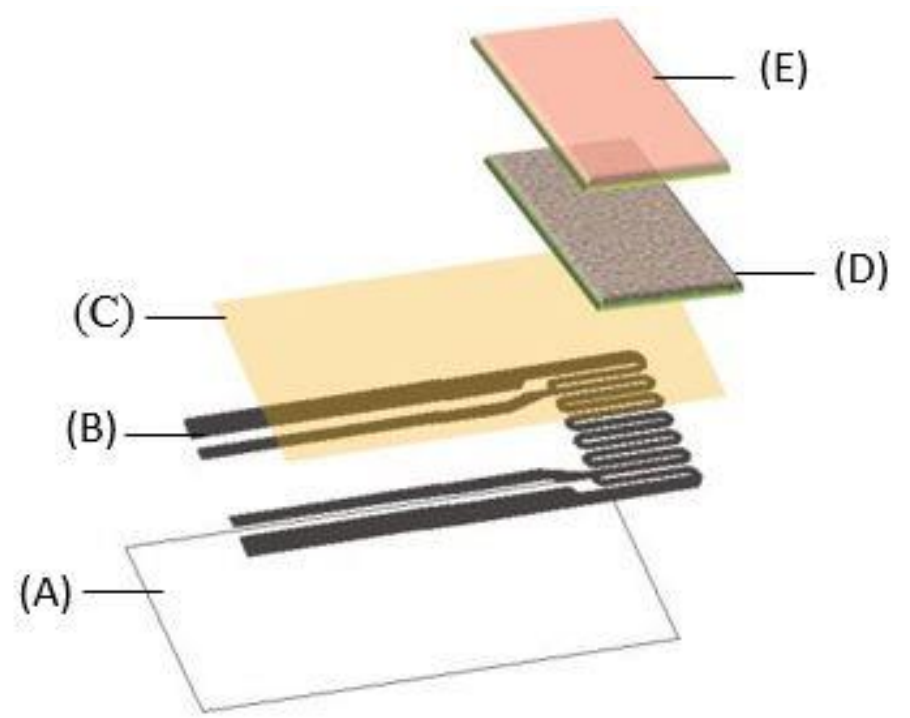

Figure 4.3 Sensor components employing oxide nanowires as a catalyst support; alumina substrate (A), Ni microheater (B), alumina oxide passivation layer (C), zinc oxide nanowires (D), sputter deposited catalyst $(\mathrm{E})$

To incorporate zinc oxide nanowires as a catalyst support, the nanowires were deposited on top of the porous alumina passivation layer and then an additional catalyst is sputter deposited onto this surface prior to experimental testing. Since previous results have shown promise for tin oxide catalyst, a $1.5 \mu \mathrm{m}$ tin oxide catalyst film was sputter deposited over the zinc oxide nanowire catalyst support.

Multiple Concentrations tests were completed using zinc oxide nanowires as the catalyst support for tin oxide, and were compared to tests using the conventional sputtered tin oxide catalyst (without the use of a support) as shown in figure 4.4 . 


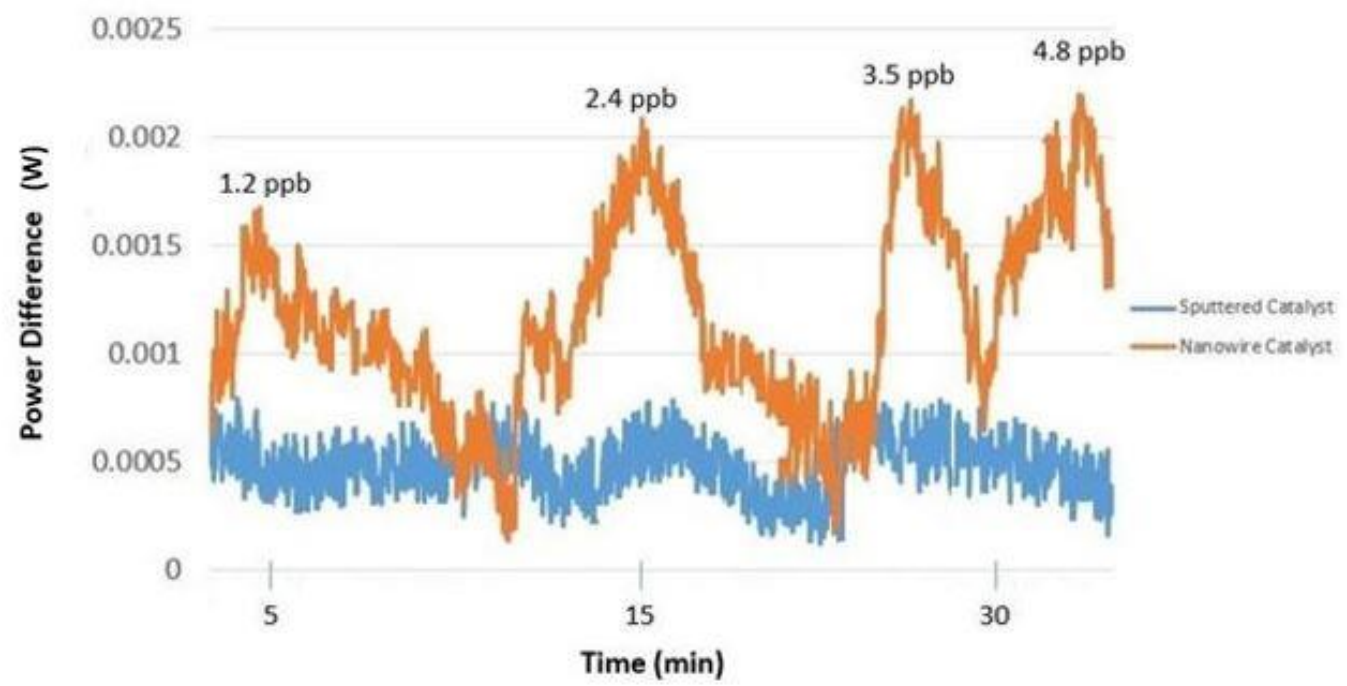

Figure 4.4 Response of two sensors with different surface area catalysts; a sputtered tin oxide catalyst (blue) and a nanowire catalyst support (red) with sputtered tin oxide catalyst, exposed to 2, 6-DNT

By employing the zinc oxide nanowires as a catalyst support, single ppb levels were achieved for 2, 6-DNT as shown figure 4.3. These were lower detection limits than seen in previous testing for 2, 6-DNT. Without the use of the zinc oxide nanowires, the sensor does not provide a reasonable signal since the signal to noise ratio is too small to decipher a response.

The catalyst support also proved to be beneficial in detecting TATP as well (figure 4.5). The signals are substantially stronger when the sensor was fabricated with nanowires as a catalyst support relative to those sensors that were fabricated using sputtered films alone, as shown in figure 4.5. 


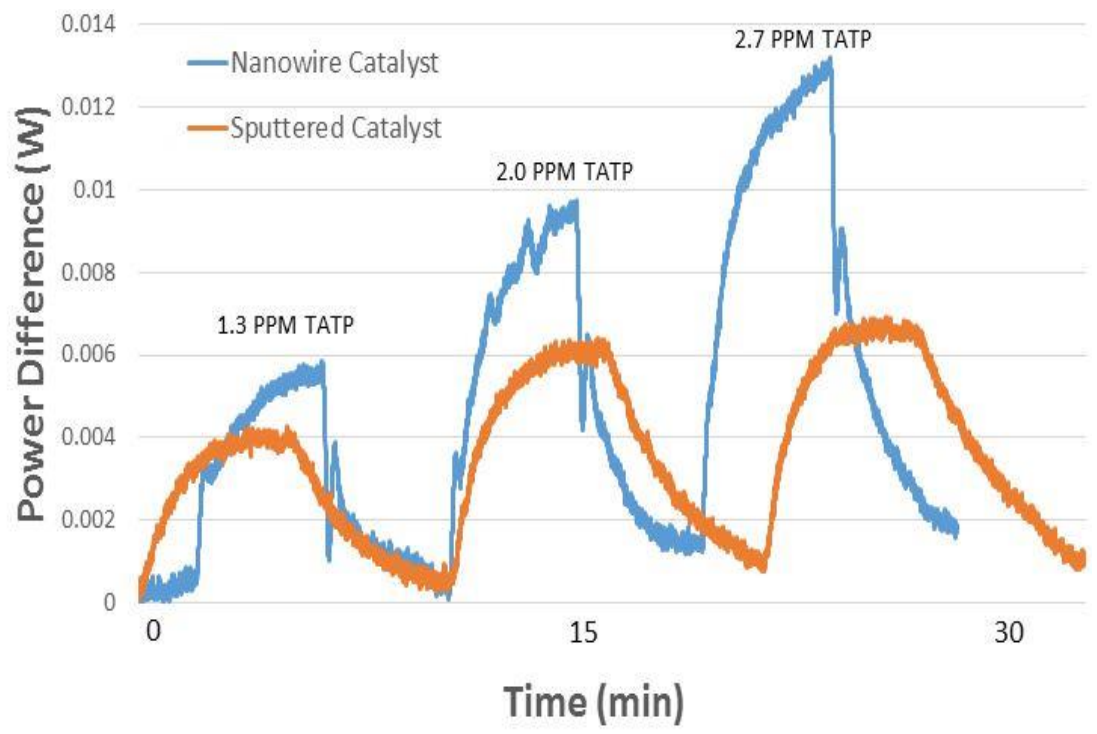

Figure 4.5 Responses of two sensors exposed to TATP; one sensor was fabricated with a nanowire catalyst support (blue) and the other with a sputtered catalyst (red) only.

At high concentrations, the signal was improved by nearly $100 \%$ when using the zinc oxide nanowires to support the tin oxide as opposed to using only the sputtered deposited tin oxide catalyst. These results speak volumes towards the merit of sensor fabrication using metal oxide nanowire catalyst supports. Not only does it improve sensitivity, but the applications can be tailored to analytes of interest.

It is clearly evident that the catalyst support has helped lower detection limits, but to better grasp how much surface area has increased, a quantitative analysis is warranted. By using figure 4.2 as a reference, quantitative stereology was used to estimate the surface area of the nanowires. We assumed that the sputtered catalysts were planar and so the surface area is simply $10000 \mu \mathrm{m}^{2}$ based on the length and width in the SEM micrograph.

When calculating the surface area of the zinc oxide nanowires, we need to be mindful that these wires cover the entire surface and so they have the same $10000 \mu \mathrm{m}^{2}$ starting point as the sputtered film. In order to calculate the surface 
area, the SEM micrograph in figure 4.2 was divided evenly into 16 individual squares. The average number of nanowires was calculated per square. The average number of zinc oxide nanowires per $1 / 16^{\text {th }}$ square was 22 wires. Using the embedded software tools in the SEM, the average diameter of a single nanowire was $4 \mu \mathrm{m}$ and the average height was determined to be $7 \mu \mathrm{m}$. Assuming that the nanowires are cylindrical in nature we can use the equation for the surface area of a cylinder to calculate the surface area of the nanowires.

$$
\mathrm{SA}(\text { cylinder })=2 \pi \mathrm{rh}+2 \pi \mathrm{r}^{2}
$$

$$
\mathrm{SA}(\text { per wire })=2 \pi(2 \mu \mathrm{m})(7 \mu \mathrm{m})+2 \pi(2 \mu \mathrm{m})^{2}
$$

Third dimensional SA $=\left(112 \mu \mathrm{m}^{2}\right)(22$ wires/square $)(16$ even squares $)$

$$
\text { Total SA }=\left(40000 \mu \mathrm{m}^{2}\right)\left(10000 \mu \mathrm{m}^{2}\right)
$$

By comparison, the zinc oxide nanowires exhibited four orders of magnitude more surface area than that provide by the sputtered catalyst. The surface area of the sputtered catalyst was calculated to be $10000 \mu \mathrm{m}^{2}$ while the nanowires were calculated to be approximately $400,000,000 \mu \mathrm{m}^{2}$ of catalyst coverage.

The zinc oxide nanowires were characterized using XRD and XPS to determine the growth mechanism by which these wires were formed. Initially, it was hypothesized that the wires grew perpendicular to the surface, growing outwards from the surface of the zinc foil, but upon further examination, proved otherwise. 


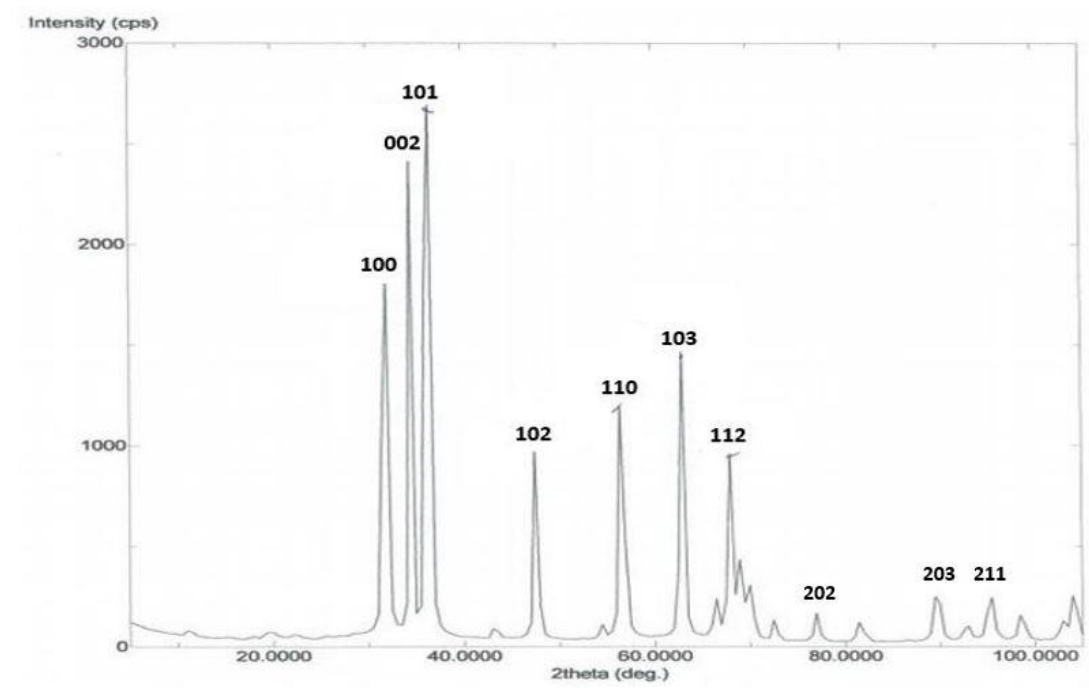

Figure 4.6 XRD pattern of zinc oxide nanowires formed on the sensor surface

XRD analysis of the zinc oxide catalyst (figure 4.6) was used to determine if any preferred orientation or texture was associated with nanowire growth. XRD analysis of the zinc oxide nanowires indicated that the oxide nanowires were largely crystalline in nature, since the relative intensities of the peaks were very similar to those from the ZnO JCPDF file [0888] [3]. XRD confirmed that as the nanowires grew, the aspect ratio became so large that the wires collapsed onto one another, forming an interconnected network of nanowires that did not yield any preferred texture. It was hypothesized that initial nanowire growth resulted in a preferred growth habit prior to collapsing, but this mechanism could not be fully supported.

XPS was used to confirm the stoichiometry of the zinc oxide nanowires (figure 4.7). 


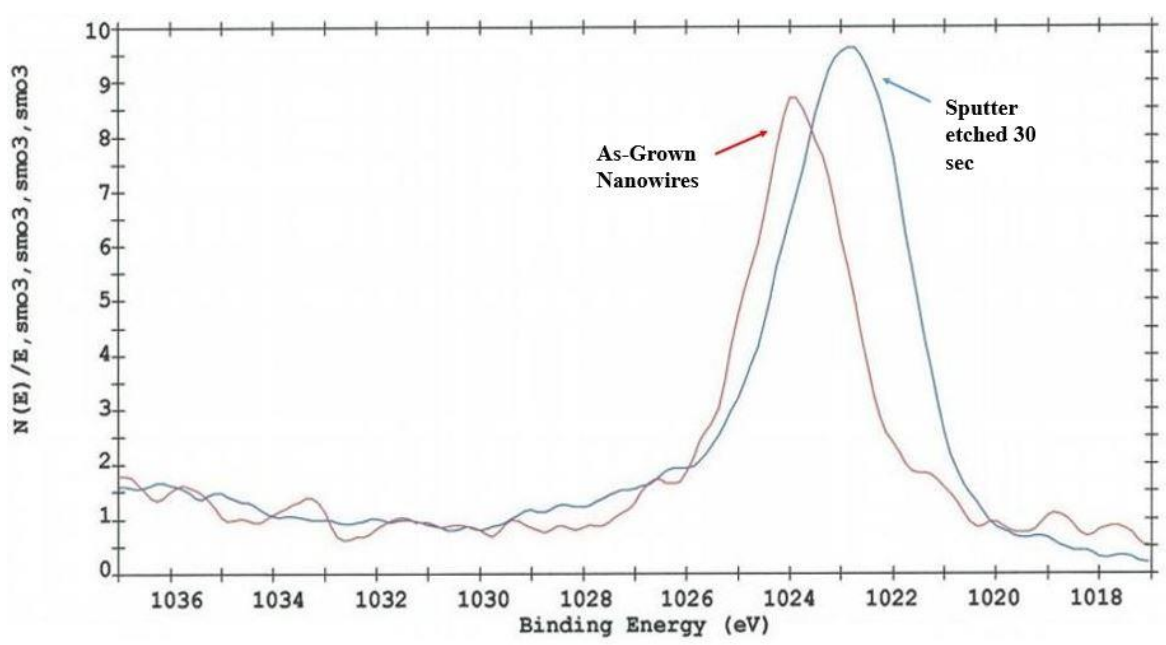

Figure 4.7 XPS spectra showing the Zn2 $\mathrm{p}^{3}$ zinc oxide peak (red) shifting to lower energy corresponding to zinc metal after 30 seconds of sputter etching (blue)

The sample was first analyzed immediately after zinc oxide nanowire growth occurred. This resulted in a binding energy of $1024 \mathrm{eV}$, which is indicative of $\mathrm{ZnO}$ [4]. Upon sputter etching for thirty seconds, an additional analysis showed a shift towards a lower binding energy of $1023 \mathrm{eV}$, which is indicative of zinc metal [4]. This indicated that the zinc metal exists in the background of the sample and that the nanowires were completely removed upon 30sec of sputter etching.

\subsubsection{Copper Oxide Nanowire Catalyst}

In terms of catalyst development, the primary goal was to develop an array of catalysts that could be tailored for specific analyte vapors. Due to its ease of fabrication, copper oxide nanowires were considered ideal candidates to enhance a library of sensor catalysts (figure 4.8). As mentioned previously, the copper oxide nanowires are grown directly on the active sensing elements of the device. 


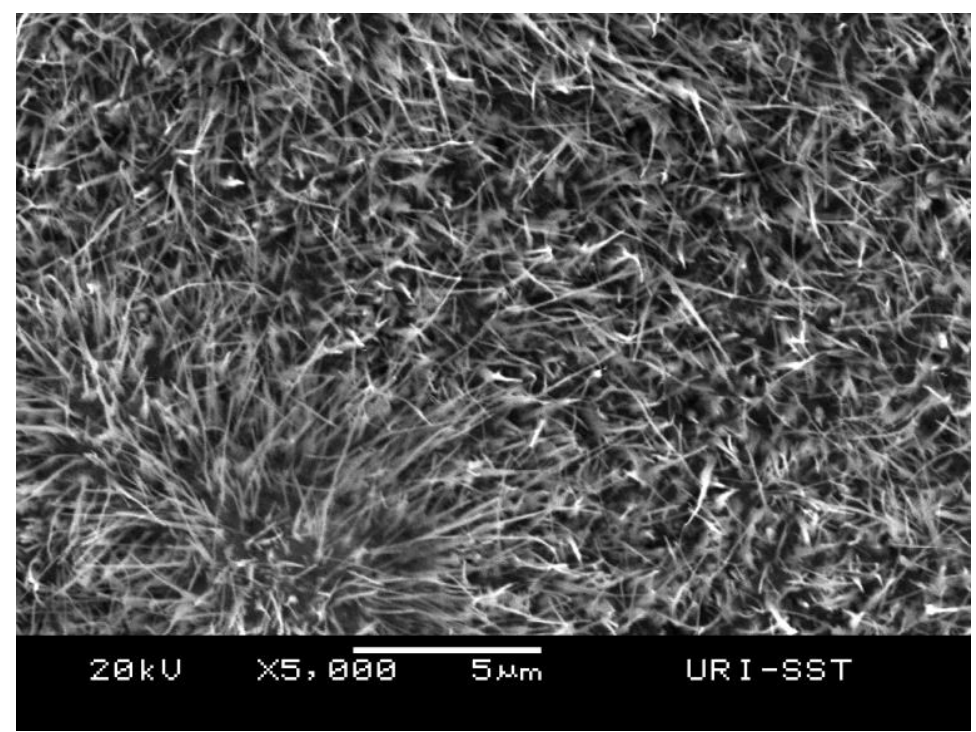

Figure 4.8 SEM micrograph of copper oxide nanowires directly grown on the thermodynamic sensor

By oxidizing the copper formed directly on the sensor surface, not only does it provide further catalytic coverage, but it also aids in reproducibility of the sensors. When manufacturing these devices, uniformity is key to ensuring that each sensor performs as well as the next. It is also important to note that the oxidation temperature for copper oxide nanowire growth has been optimized at 400C and the resulting morphology is shown in figure 4.8. Upon lowering the temperature to $340 \mathrm{C}$, the nanowires do not produce the same coverage of the surface as seen in figure 4.9 . 


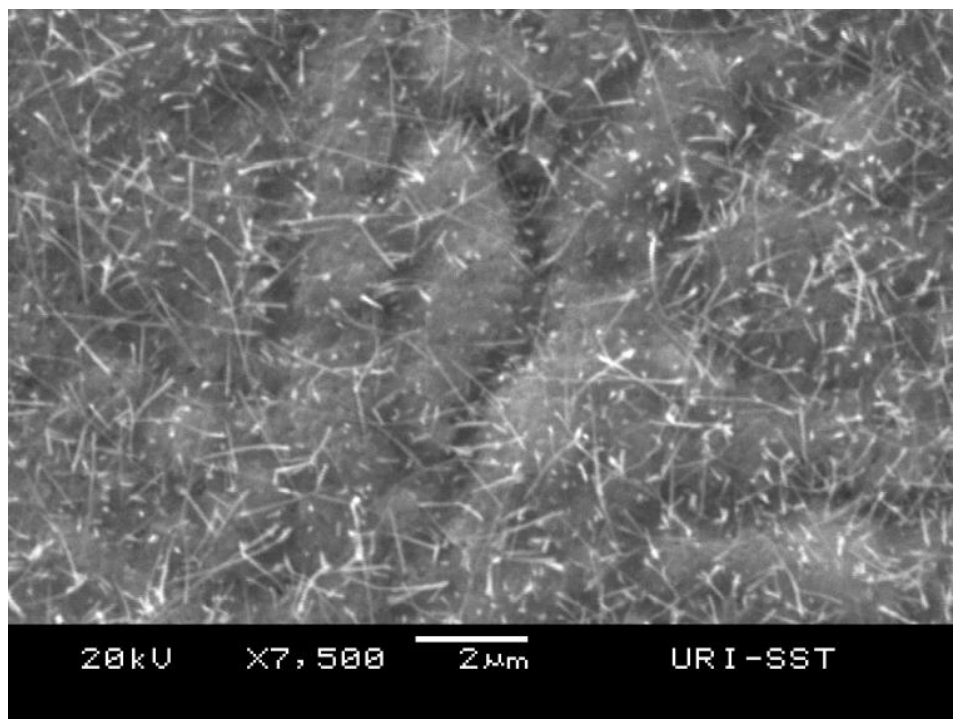

Figure 4.9 SEM micrograph of copper oxide nanowires thermally grown at 340C for 5hours Decreasing the oxidation temperature to 340C affected the growth of the nanowires dramatically. Not only do we see a microstructure that provides less coverage, but we also expose the underlying copper metal deposited film.

To analyze the growth of the copper oxide nanowires, XRD and XPS was again used to characterize the material. Similar to that of the zinc oxide nanowires, the growth of copper oxide nanowires exhibited little or no preferred growth (figure 4.10).

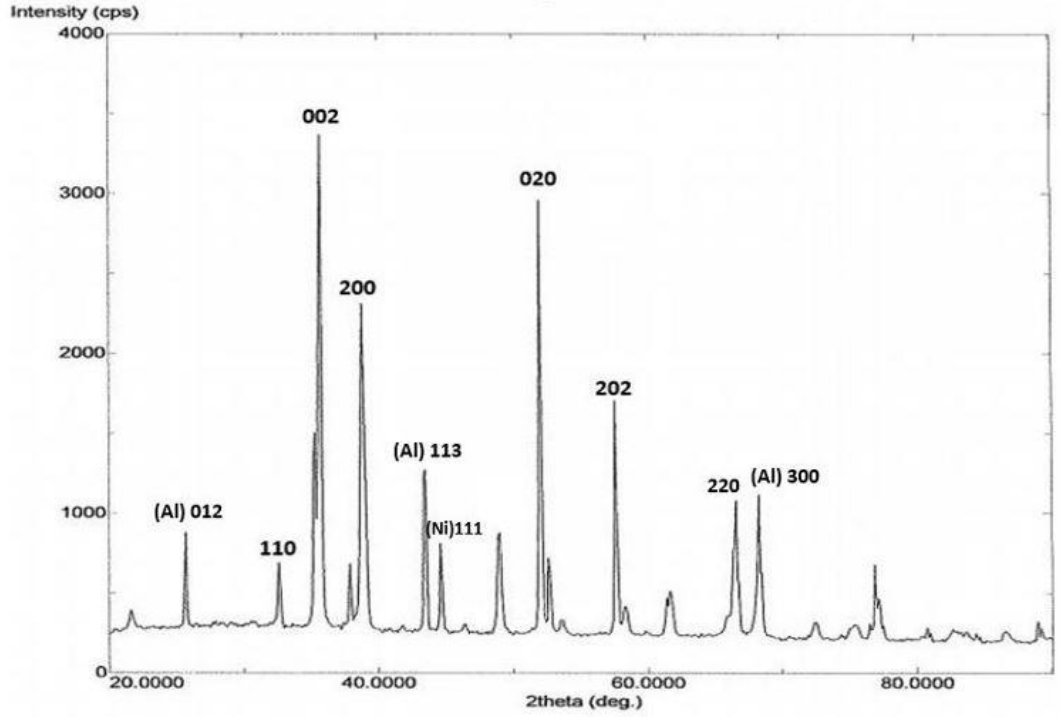

Figure 4.10 XRD pattern of the copper oxide nanowire catalyst 
The XRD pattern of the copper oxide nanowires indicated that nanowires were largely crystalline in nature with no preferred orientation. Interestingly enough, peaks due to background material resulting from the fabrication method are also shown [3]. These peaks were identified as aluminum oxide and nickel were attributed to the penetration of the beam into the underlying layers. The XPS spectra of the copper oxide nanowire catalyst revealed results similar to those of zinc oxide as shown in figure 4.11 .

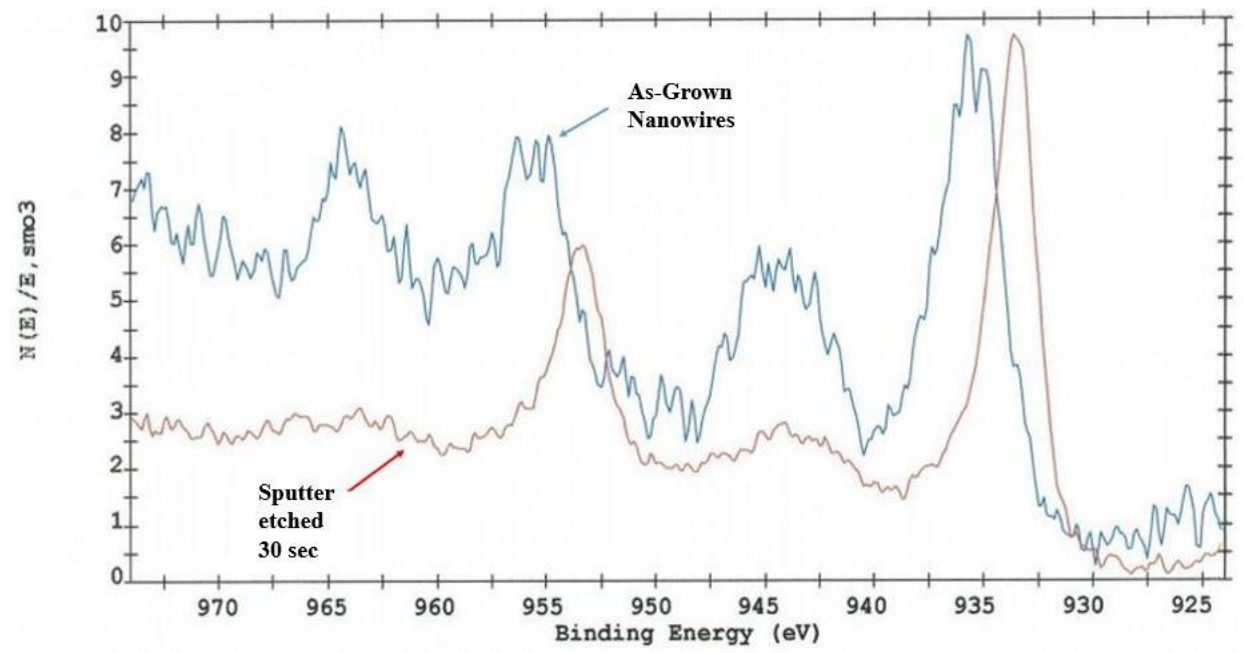

Figure 4.11 XPS analysis of the copper oxide nanowire catalyst

The copper oxide nanowire catalyst was initially analyzed by XPS and after 30 seconds of sputter etching. During the initial XPS scan, four peaks were identified, which signify the presence of $\mathrm{CuO}$ [4]. After 30 seconds of sputter etching, however, the $\mathrm{Cu} 2 \mathrm{p}^{1}$ and $\mathrm{Cu} 2 \mathrm{p}^{3}$ peaks shifted to lower energy and the two peaks at $965 \mathrm{eV}$ and $945 \mathrm{eV}$ disappeared, confirming that the $\mathrm{CuO}$ nanowires were removed by etching, leaving behind metallic copper from which the nanowires were grown [4].

\subsubsection{Iron Oxide Nanowire Catalyst}


A third catalyst was added to the catalyst library developed for the solidstate sensors. Iron oxide was selected as a catalyst since iron possesses many of the same characteristics as copper and zinc in that its oxidation state can be manipulated for a specific reaction. Along those lines, direct fabrication can be achieved using controlled thermal oxidation, (figure 4.12).

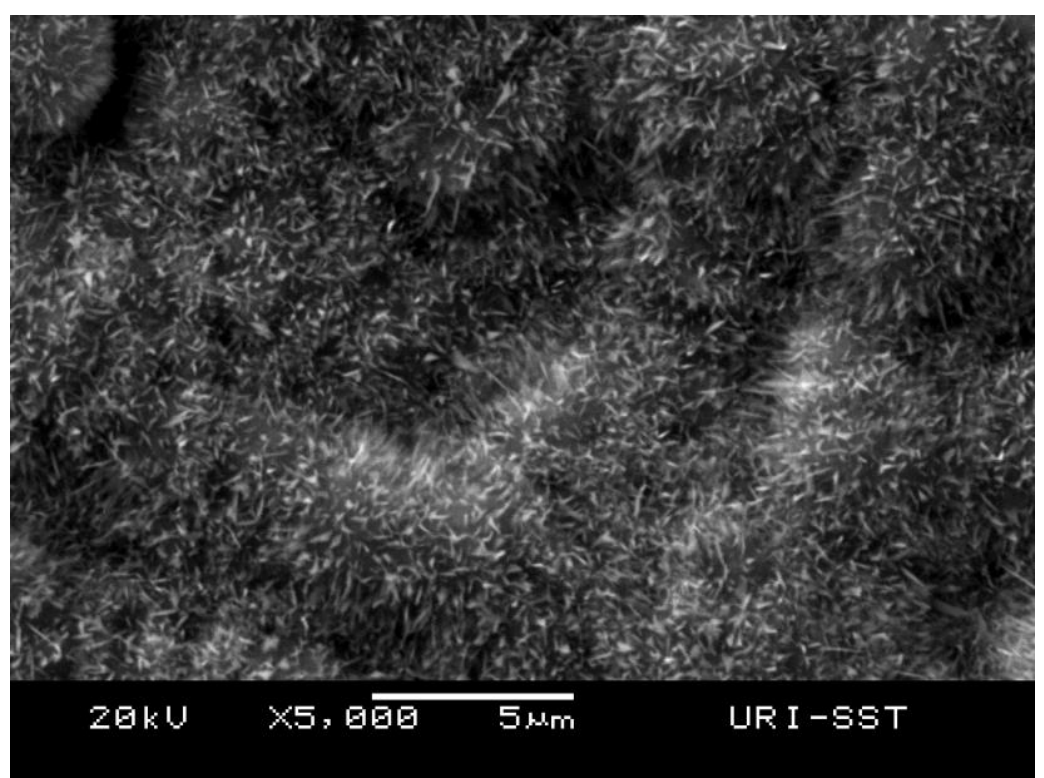

Figure 4.12 SEM micrograph of iron oxide nanowires thermally grown on the serpentine of the microheater

Complete coverage of iron oxide nanowires over the microheater surface was achieved and was equivalent to that of the copper oxide coverage, which exhibits the best behavior to date in terms of sensor performance enhancement. Elevated oxidizing temperatures were also investigated to see its effect it has on surface coverage (figure 4.13). 


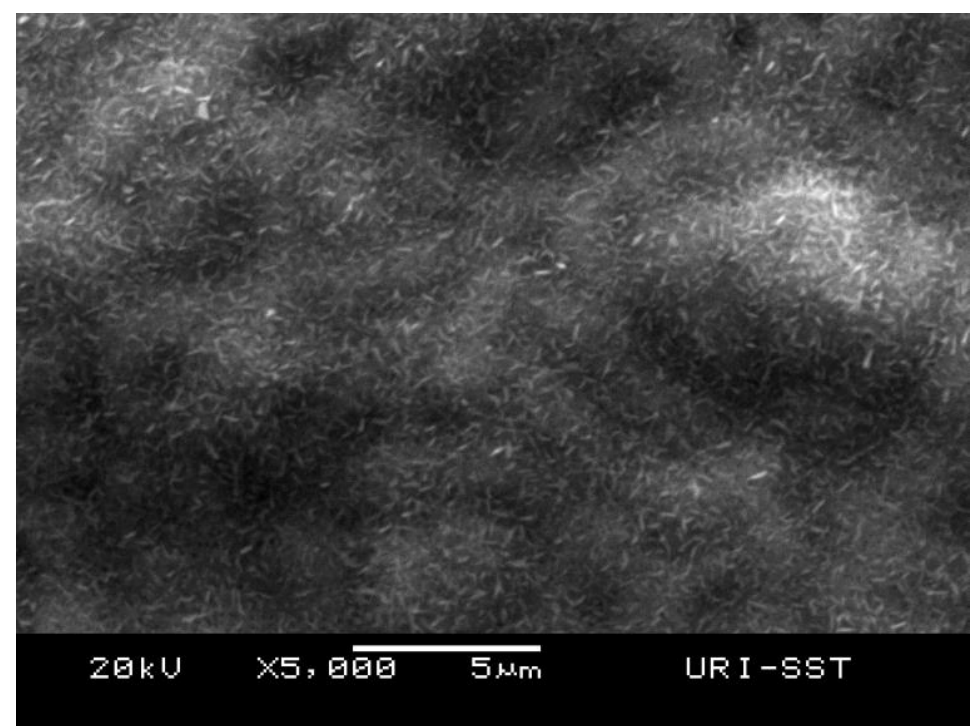

Figure 4.13 SEM micrograph of iron oxide nanowire grown at 600C

It appears that the nanowires are not as well defined at elevated temperatures and thus, less than desirable surfaces were obtained when compared to growth at 400C [6]. The mechanism responsible for iron oxide and copper oxide nanowire growth is attributed to convective heating caused by evaporation and redeposition onto the surface. In this manner, as the oxide particles are re-deposited onto the sensor surface, they build upon each other causing a nanowire structure to develop. When the correct temperature is reached, the evaporation and the redeposition rates are optimized and nanowire growth results on the surface.

XRD (figure 4.14) and XPS (figure 4.15) was used to characterize the crystallinity of the nanowires and resulted in slightly different results when compared to the zinc oxide nanowires and copper oxide nanowires. 


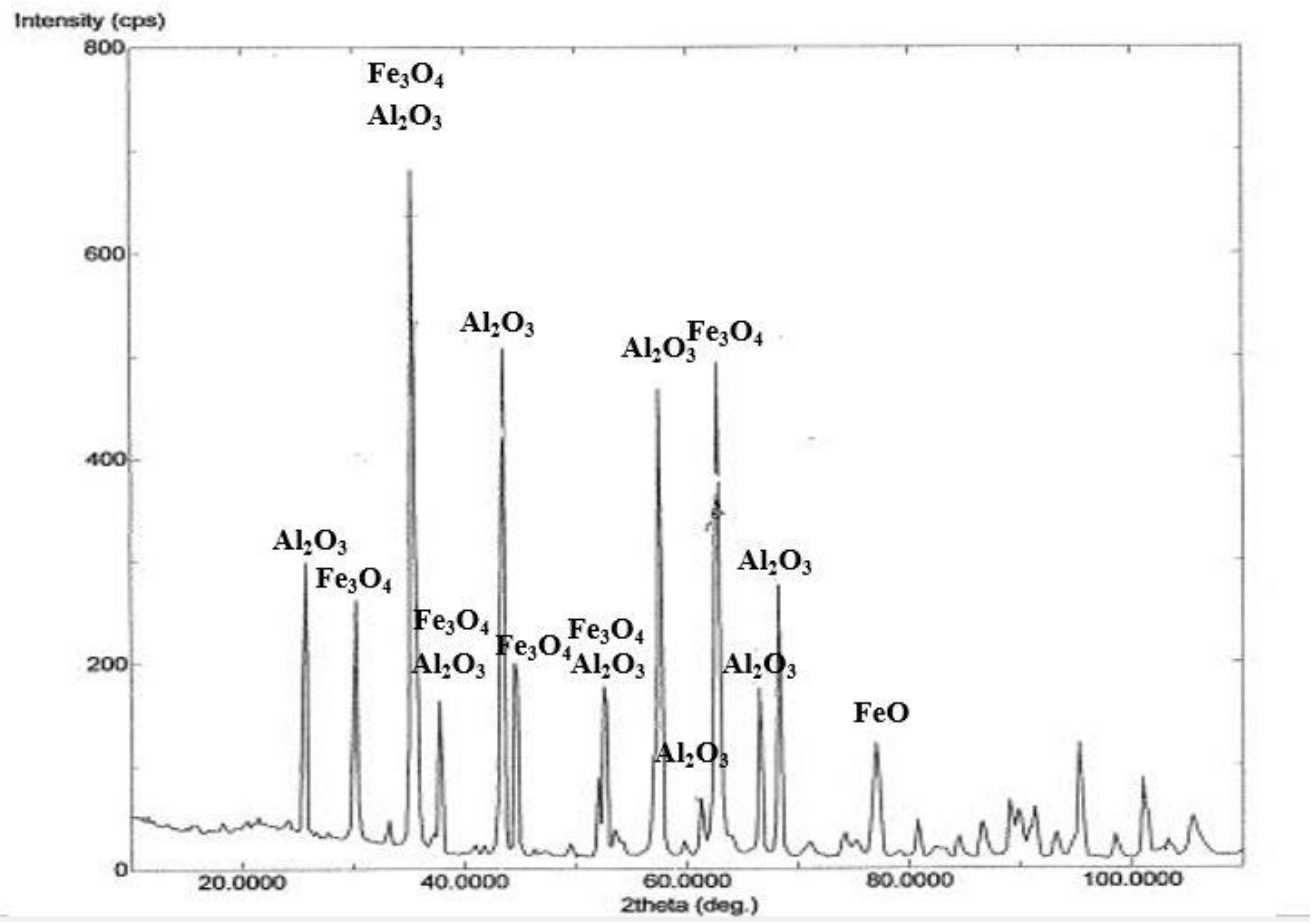

Figure 4.14 XRD pattern of the thermally grown iron oxide nanowires

The XRD pattern suggests a combination of $\mathrm{FeO}$ and $\mathrm{Fe}_{2} \mathrm{O}_{3}$ formed on the surface as a result of thermal oxidation [3]. Contribution from the aluminum oxide passivation layer is evident in the pattern as well as contribution from $\mathrm{FeO}$ [3]. These additional contributions caused a shift in peak intensities and $2 \theta$ angles when the magnetite phase $\left(\mathrm{Fe}_{3} \mathrm{O}_{4}\right)$ of iron oxide is formed, i.e. a mixture of $\mathrm{FeO}$ (rich) salt and $\mathrm{Fe}_{2} \mathrm{O}_{3}$ (hematite). 


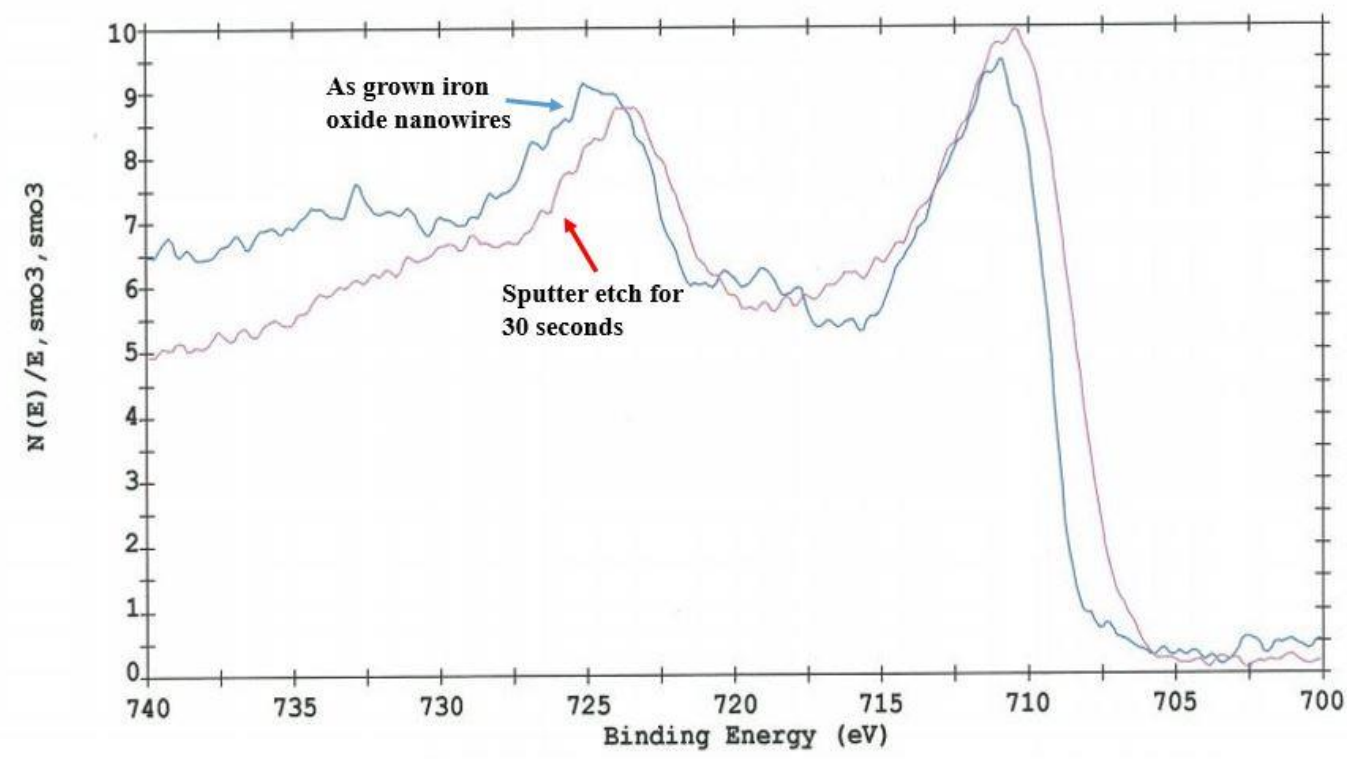

Figure 4.15 XPS overlay plot of iron oxide nanowires prior to and after sputter etching

A peak shift in the XPS spectrum was observed after sputter etching, confirming that the iron oxide nanowires were completely removed after 30 seconds of etching. The XPS spectrum of the iron oxide nanowires is shown in (figure 4.16).

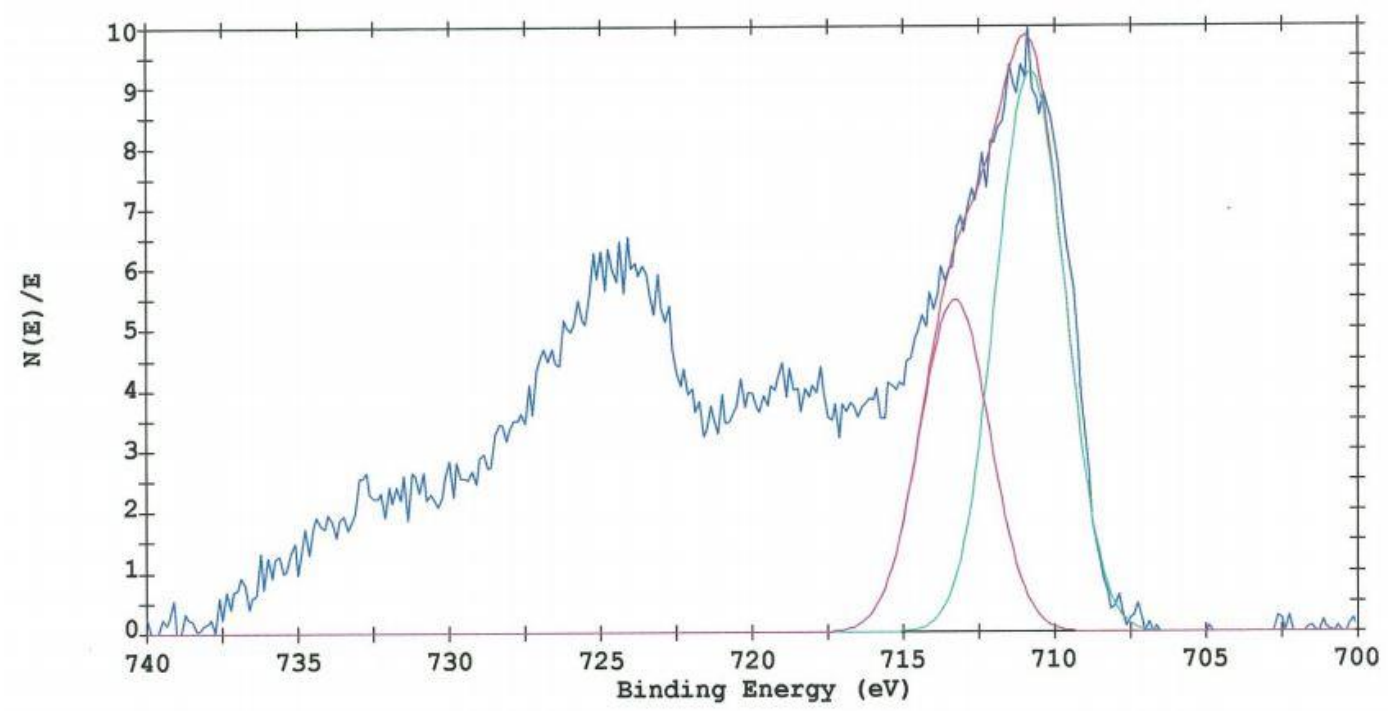

Figure 4.16 XPS analysis of the iron oxide nanowires

The main peak at $712 \mathrm{eV}$ in the XPS spectra was much broader than had been observed in the literature [4]. This coincides with the fact that the XRD pattern indicated a multi-component system of $\mathrm{FeO}$ and $\mathrm{Fe}_{2} \mathrm{O}_{3}$ and oxidizes to form 
magnetite. Two peaks were observed in figure 4.16 which indicate the relative contributions to the peak broadening that was observed. Further testing will be completed to determine the effects of this mixture of oxides and their response to various analytes.

\subsection{Sensor Design that Targets Explosives}

One of the priorities associated with this sensing platform is mitigating false positives. The only way that this sensor can be sued in a field trial, from a manufacturing standpoint, is if it can specifically target analytes without false positives. It has to be able to sense energetic materials passively, in an uncontrolled environment, and in the presence of other chemicals. Interferences are used to try to "trick" the sensor and terrorists could use this to their advantage for attack. This would prevent explosive detection until the bomb is set in place and makes this type of data invaluable.

In order to demonstrate specificity of the sensor, we intentionally introduced compounds that are commonly found in cargo containers (figure 4.17), which show the effect of interference on the sensor performance.

\section{TATP with Methyl Acrylate}

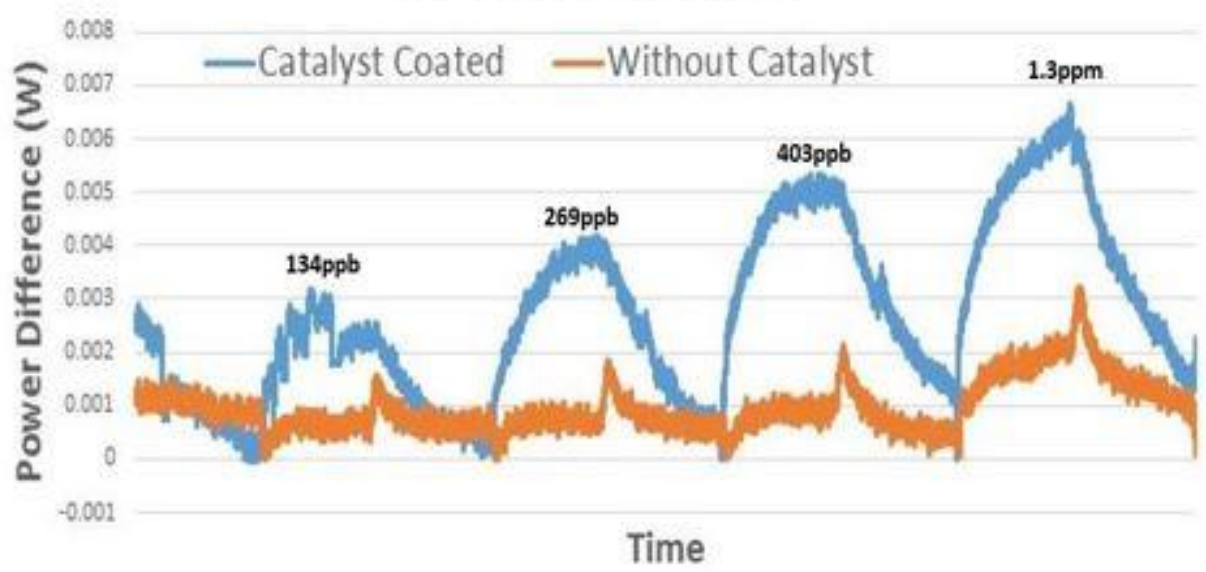

Figure 4.17 Sensor response to TATP in the presence of methyl acrylate 
Sensor responses were measured for TATP in the presence of an adhesive that is commonly found in cargo containers. Methyl acrylate for example, is one of the chemicals found in the production of packing tape, which was thought to potentially mask the analytes from the detector. Not only that, but methyl acrylate has a much higher vapor pressure than the energetic materials being tested and thus, would be present at higher concentration in the vapor phase. Based on the results, the sensor could still detect the TATP in the presence of methyl acrylate. Response curves were similar those that we have seen in previous exposures and the power change was also similar in magnitude. Since there is a much higher concentration of acrylate in the vapor phase than TATP, we would have expected to see a diminished response to TATP with the acrylate masking the response. This speaks volumes to how important tailoring the catalyst is for the detection of energetic materials, and adds to the robust nature of our gas sensor. 


\section{REFERENCES}

[1] Yajun J. One-step method for growing of large scale $\mathrm{ZnO}$ nanowires on zinc foil. Materials Letters 138 (2015): 92-95.

[2] Baratto C., Kumar R., Comini E., Faglia G., Sberveglieri G. Gas sensing study $\underline{\text { of } \mathrm{ZnO} \text { nanowire heterostructured with } \mathrm{NiO} \text { for detection of pollutant }}$ gases. Procedia Enginnering vol. 87 (2014): 1091-1094

[3] Cullity B. D. and Stock S. R. Elements of X-Ray Diffraction. Prentice Hall (2001) ed. $3^{\text {rd }}$

[4] Moulder J. F., Stickle W. F., Sobol P. E., Bomben K. D. Handbook of X-ray Photoelectron Spectroscopy. ULVAC-PHI Inc. (1995).

[5] Chopra N., Shi W. and Lattner A. Fabrication and characterization of copper $\underline{\text { oxide }(\mathrm{CuO}) \text {-gold }(\mathrm{Au}) \text {-titania }(\mathrm{TiO} 2) \text { and copper oxide }(\mathrm{CuO}) \text {-gold }}$ (Au)-indium tin oxide (ITO) nanowire heterostructures. Materials Characterization 96 (2014): 71-77.

[6] Shen X., Song F., Yang X., Wang Z., Jing M., Wang Y., Hexaferrite/ $\alpha$-iron composite nanowires: Microstructure, exchange-coupling interaction and microwave absorption. Journal of Alloys and Compounds 621 (2015): $146-153$ 


\section{CHAPTER 5}

\section{CONCLUSION}

\subsection{Conclusion}

The objective of this research was to improve the selectivity and sensitivity of the solid-state thermodynamic gas sensor for trace explosive detection. These improvements would greatly assist with the transition to a MEMS based platform. One avenue to exploit was to effectively increase the surface area available for the catalytic decomposition of energetic materials. This research focused on fabricating catalysts in such a way that they could be useful for the existing sensor platform and the MEMS platform.

Metal oxide nanowires were then explored as catalysts and catalysts support to increase signal response without sacrificing sensor real estate. Because of their ease of fabrication as well as multiple oxidation states, zinc oxide, copper oxide and iron oxide nanowires were employed as the catalyst for the gas sensors. Zinc oxide nanowires were hydrothermally grown on pure zinc and deposited onto sensors as a catalyst support while copper and iron oxide nanowires were grown directly on the sensing elements via thermal oxidation.

All of the nanowires exhibited complete coverage of the serpentine of the microheater, which resulted in dramatic increase in signal strength when used to detect explosives. This improved the minimum detection limit for threat molecules using this gas detection system (table 5.1). 


\begin{tabular}{|c|c|c|}
\hline Analyte & $\begin{array}{c}\text { Previous Detection } \\
\text { Limit }\end{array}$ & New Detection Limit \\
\hline TATP & $200 \mathrm{ppb}$ & $100 \mathrm{ppb}$ \\
\hline $2,6-\mathrm{DNT}$ & $50 \mathrm{ppb}$ & $500 \mathrm{ppt}$ \\
\hline
\end{tabular}

Table 5.1 Detection limits for threat molecules using the zinc oxide nanowire catalyst support

XRD and XPS analysis showed some similarities between the various nanowires in that they were largely crystalline in nature and they tend not to grow with a preferred orientation. One major difference between the various nanowires is that the mechanism by which iron oxide nanowires are grown, and produce multiple oxidation states of iron is provided by a combination of oxides, supported by XRD and XPS of the various oxides of iron. XPS spectra showed similar characteristics for each catalyst. A shift in peaks or the disappearance of secondary peaks were observed and indicated that after 30 seconds of sputter etching, the nanowires were completely removed from the surface, exposing the underlying metal.

Lastly, specificity was demonstrated by testing for potential chemicals that could be used for masking the response towards energetic materials. The thermodynamic sensor proved to discriminate between other molecules in the vapor phase, yielding a signal only due to that of TATP. Further investigation into other potential masking agents will help to prove how valuable this sensor can be to passively monitor high traffic areas and protect the travelling public.

\subsection{Future Work}

As research continues, there are some areas, which can be explored further to improve the gas sensor. With a decrease in detection limits as well as better 
lithographic techniques, a transition towards a MEMS device may be in the very near future.

\subsubsection{Conductometric Sensor Applications}

As mentioned previously, the novel aspect of this gas sensor is that it has built-in orthogonality; i.e. the sensor has a thermodynamic platform along with a conductometric platform from which these signals lead to a built-in redundancy. The conductometric response results from changes in the resistivity of the metal oxide catalyst upon exposure to threat molecules. By using this additional response, not only will it improve sensitivity, but it will also help to mitigate false positives in the presence of a false thermodynamic response. Theoretically, by using metal oxide nanowires, the additional catalytic interactions should result in a larger change in resistivity to be measured. Further research will be conducted to see how metal oxide nanowire catalysts affect the resistivity response.

\subsubsection{Potential Analytes}

The practicality of the thermodynamic sensor allows for many applications. One application that is gaining headway is that monitoring of imported goods. Because of the potential size of the gas sensor, it can be used to closely monitor cargo as it passes through major ports in the U.S. One of the biggest concerns is preventing drugs from being smuggled into the country. Since these drugs have a given vapor pressure, there is possibility for decomposition when adhering to the sensor surface. It would be ideal to test various drugs such as methamphetamines and other drugs that are highly touted in trades. Testing would be similar to that of TATP, where the drugs are made in a solution that can 
be applied to filter paper and loaded in the "target chamber" of the dynamic testing setup. As long as these drugs can cause a power change, then this would be another application for the robust sensor. Not only that, but other gases such as ammonia could be tested which helps to build the list of applications for the device. Ammonia levels are important in agriculture and so there may be a large market for such a sensor.

\subsubsection{Transition towards Environmental Testing}

All of the data acquired thus far has been completed in a controlled laboratory environment to ensure validity of the sensors. In order to move towards a commercial device, it should be field tested under various humidity and temperature conditions. With this in mind, we have developed a portable testing system (figure 5.1).

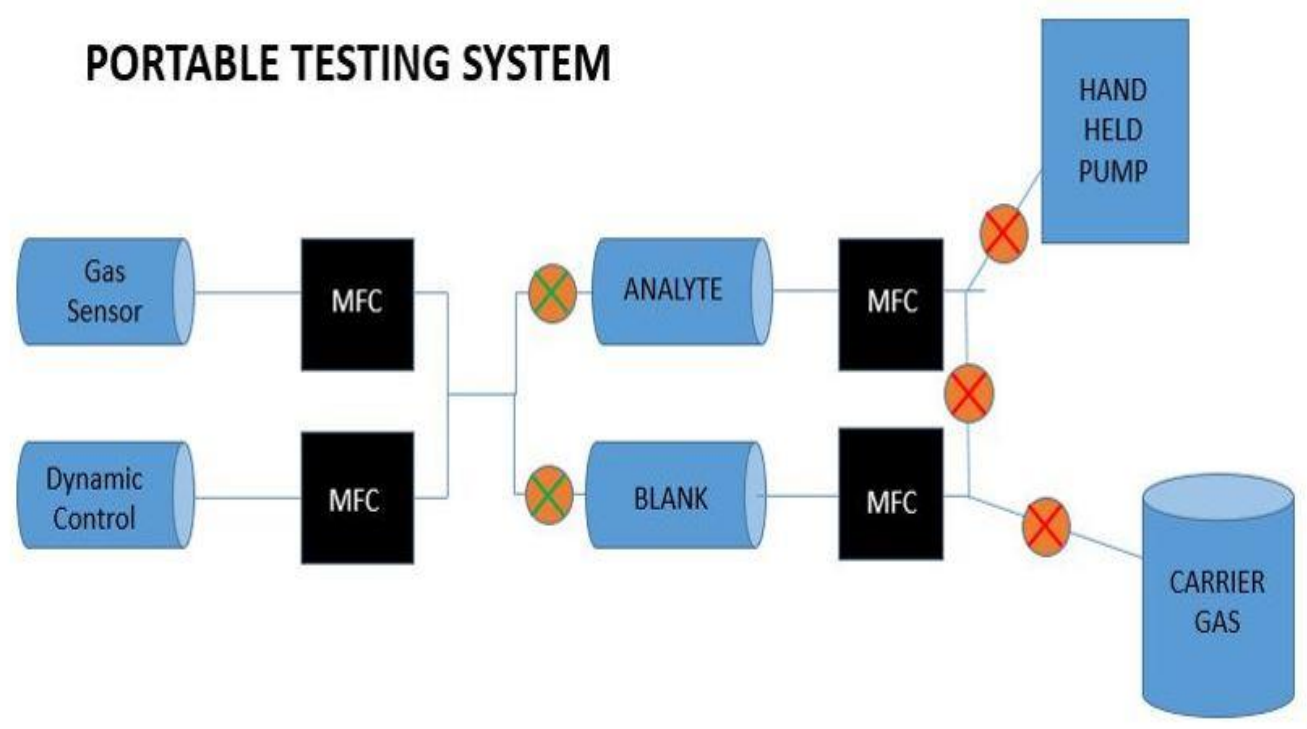

Figure 5.1 Portable testing system for field applications

The portable system is similar to that of the dynamic control experimental setup with the addition of a hand held pump. By creating a setup with additional valves 
and a hand held pump, not only can we sample the air for testing but we can transport it into various climates and adjust the concentration ourselves with the carrier gas attachment. The setup will again use four mass flow controllers so that concentrations of analyte may be varied and the entire apparatus would be interfaced to a laptop running the data acquisition system. Measurements and stainless steel piping have already been assessed and are ready to be assembled as soon as the remaining parts arrive. From there, the system will be assembled and field testing can proceed.

\subsubsection{Pre-concentration Improvement}

Pre-concentration has been explored as a possibility to decrease minimum detection limits, by delivering a concentrated burst of analytes to the sensor. Referring to table 2.1, polystyrene has been the leading candidate for preconcentration and although there has been proven success, these polymers present some issues. Polystyrene has a high affinity toward nitrogen containing compounds and has lowered detection limits for analytes such as 2, 6-DNT but will this translate to other analytes of interest? By using a polymer that only adsorbs nitrogen containing analytes, the sensor becomes less attractive from a broad based detection standpoint. Not only that, but because the polymer is porous in nature, the desorption kinetics become much more complicated and hence the $58 \%$ desorption rate. By increasing this rate, detection limits can be lowered.

In order to further improve pre-concentration, a new method should be explored that is being highly touted in the medical field currently. The method is 
called photo-chemotherapy. Chemotherapy drugs are known to be highly reactive in the body, which is the reason why patients develop side effects such as hair loss and damage to healthy cells. The latest development in chemotherapy has been the use of light to activate drugs. The idea is that the drug is attached to some sort of metal complex causing the drug to be inactive. As the drug moves through the body, exposure to infrared light, activates the metal complex and releases the drug. For the patient, this means localized treatment and a reduction in damage to nearby healthy cells.

This same premise can be used for pre-concentration; but instead of coating an additional microheater with a polymer, the sensor would be coated with a light activated metal complex that has a high affinity for energetic compounds. Periodically, a $\mathrm{CO}_{2}$ laser could be used to activate the complex, releasing the analytes and delivering them to the active sensor element. There would be no issue of porosity and adsorption/desorption kinetics. It is known that the reaction will take place fully upon activation and thus, $100 \%$ desorption would take place. Not only would this improve detection limits, but the complex may be easy to tailor for specific analytes of interest.

\subsubsection{MEMS Device}

Great strides have been made in the transition towards a MEMS based platform that has been greatly influenced by this research. Nanowires have proven not only to be easily fabricated, but also to lower detection limits for threat molecules. That being said, future MEMS devices will employ metal oxide nanowire catalysts (figure 5.2). 


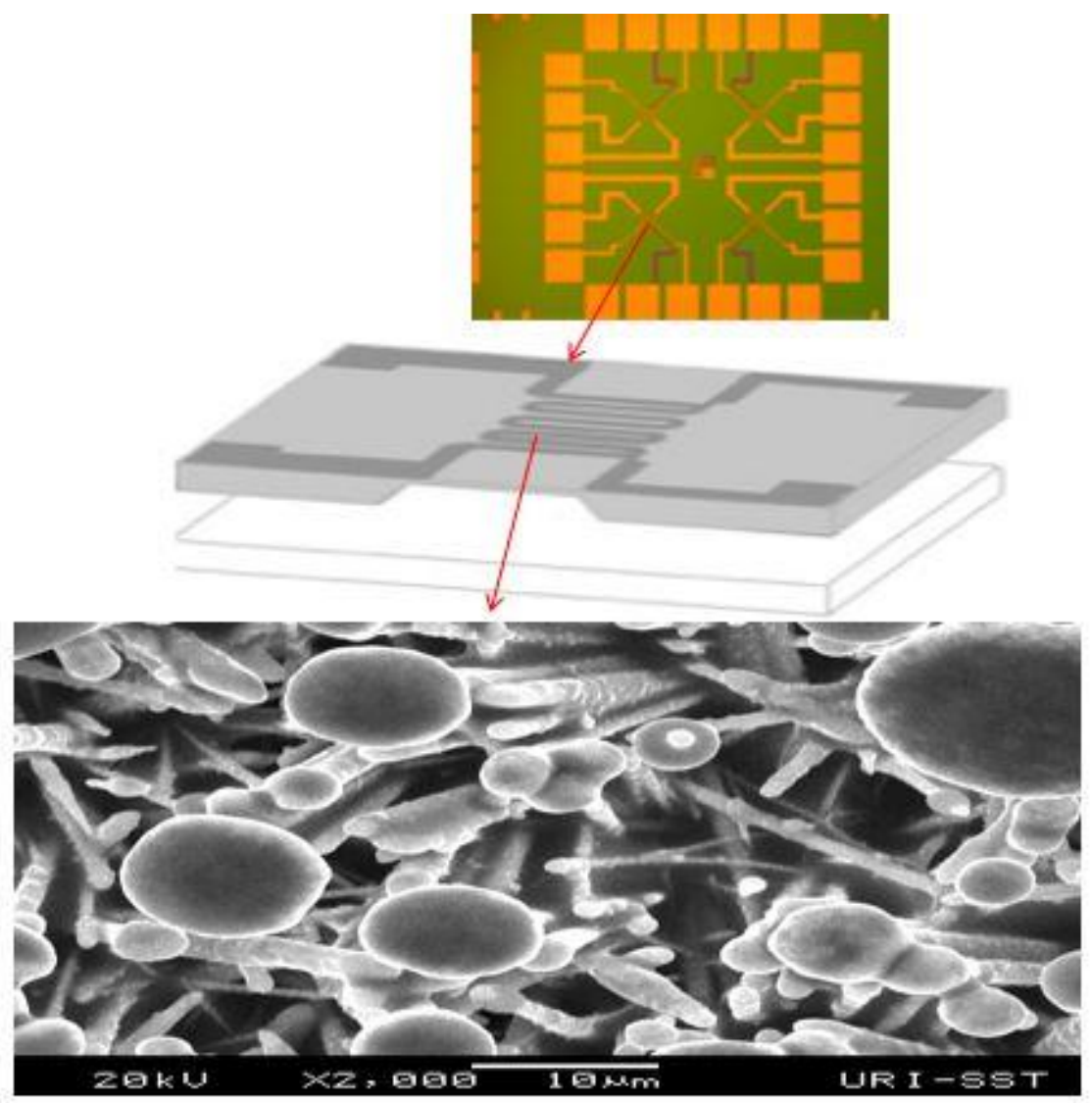

Figure 5.2 Applications for metal oxide nanowire catalysts on MEMS based devices

As one of the final steps in the four-quadrant device, metal oxide nanowires can be thermally grown to enhance the sensitivity and selectivity of the sensor. It is theorized that various nanowires can be fabricated onto each of the quadrants to specifically target different threats. These key improvements along with others will eventually translate into a more robust, manufactured sensor. 


\section{BIBLIOGRAPHY}

Agrawal J. P., Hodgson R. D., Organic Chemistry of Explosives. John Wiley \& Sons (2007)

Aguilar A. D., Forzani E. S., Leright M., Tsow F., Cagan A., Iglesias R. A., Nagahara L. A., Amiani I., Tsui R. and Tao N. J. A Hybrid Nanosensor for TNT Vapor Detection. Nanoletters 10 (2010): 380-384

Albert K. J., Myrick M. L., Brown S. B., James D. L., Milanovich F. P., Walt D. R. Field- Deployable Sniffer for 2, 4-dinitrotoluene Detection. Environ. Sci. Technol 35 (2001): 3193.

Archibong J. Ituh. Port Security Technology for Closed Container Inspection at United States Seaports of Entry University of Oregon 2010.

Arshak K., Moore E., Lyons G. M., Harris J. and Clifford S. A Review of Gas

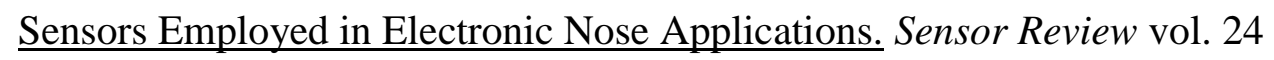
(2004): 181-198

Barbero, Michael D. Improvised Explosive Devices Are Here to Stay. The Washington Post. N.p., 18 May 2013. Web. 6 Sept. 2014.

Baratto C., Kumar R., Comini E., Faglia G., Sberveglieri G. Gas sensing study of $\underline{\mathrm{ZnO} \text { nanowire heterostructured with } \mathrm{NiO} \text { for detection of pollutant gases. }}$ Procedia Enginnering vol. 87 (2014): 1091-1094

Berchtold C., Meier L. and Zenobi R. Evaluation of extractive electrospray $\underline{\text { ionization and atmospheric pressure chemical ionization for the detection }}$ of narcotics in breath. International Journal of Mass Spectrometry 299.2-3 
(2011): 145-150.

Brady J. E., Smith J. L., Hart C. E. and Oxley J. Estimating Ambient Vapor Pressures of Low Volatility Explosives by Rising Temperature Thermogravimetry. Propellants Explos. Pyrotech. (2012): 215-222.

Camara M., James F., Breuil P., Pijolat C., Briand D., de Rooij N. F., MEMSbased porous silicon preconcentrators filled with Carbopack-B for explosives detection. Procedia Engineering vol. 87 (2014): 84-87.

Cell Press. New technique for precise light-activated chemotherapy drugs. Science Daily (2015) accessed: March 2016 $<$ www.sciencedaily.com/releases/2015/07/150709132433.htm>

Chopra N., Shi W. and Lattner A. Fabrication and characterization of copper oxide $(\mathrm{CuO})$-gold $(\mathrm{Au})$-titania $(\mathrm{TiO} 2)$ and copper oxide $(\mathrm{CuO})$-gold

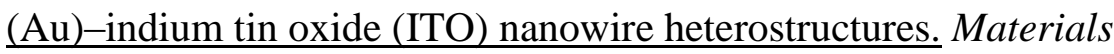
Characterization 96 (2014): 71-77.

Chuang H., Hong G., Sanchez J. Fabrication of high aspect ratio copper $\underline{\text { nanowires using supercritical } \mathrm{CO}_{2} \text { fluids electroplating technique in } \mathrm{AAO}}$ template. Materials Science in Semiconductor Processing 45 (2016): 1726

Cooks R. G., Ouyang Z., Takats Z., Wiseman J. M. Detection Technologies. Ambient Mass Spectrometry. Science 311 (2006): 1566-1570.

Cullity B. D. and Stock S. R. Elements of X-Ray Diffraction. Prentice Hall (2001) ed. $3^{\text {rd }}$ 
Ewing R. A critical review of ion mobility spectrometry for the detection of explosives and explosive related compounds. Talanta 54.3 (2001): $515-$ 529.

Ewing R. G., Atkinson D. A., Clowers B. H., Direct Real-Time Detection of RDX Vapors Under Ambient Conditions Analytical Chemistry 85 (2013)

Furton K. The scientific foundation and efficacy of the use of canines as chemical detectors for explosives. Talanta 54.3 (2001): 487-500.

Gazit I. and Terkel J. Explosives detection by sniffer dogs following strenuous physical activity. Applied Animal Behaviour Science 81.2 (2003): 149161.

Hill R. J. Physical Vapor Deposition. Temescal (1986) ed. $2^{\text {nd }}$

Hussein E. and Waller E. Review of one-side approaches to radiographic imaging for detection of explosives and narcotics. Radiation Measurements 29.6 (1998): 581-591.

Jamison J. Canine Drug Program Cost Analysis. Idaho Criminal Justice Council (2004).

Lesniak A., Walczak M., Jezierski T., Sacharczuk M., Gawkowski M., Jaszczak K. Canine Olfactory Receptor Gene Polymorphism and its Relation to Odor Detection Performance by Sniffer Dogs. J. Hered 99 (2008): 518 527.

Mallin D. Increasing the Selectivity and Sensitivity of Gas Sensors for the Detection of Explosives. University of Rhode Island M.S. thesis (2014). 
Martin M., Crain M., Walsh K., McGill R. et al. Microfabricated Vapor Preconcen. for Port. Ion Mobility Spect. Sensors and Actuators B 126 (2007): 447-454.

Mason S. Let there be tissue-penetrating light: Scientists develop new nanoscale method to fight cancer. UCLA Newsroom (2014).

Miller J. S., Johansen R. T. Fracturing Oil Shale with Explosives for In Situ Recovery. American Chemical Society 151 Retrieved (2015)

Moulder J. F., Stickle W. F., Sobol P. E., Bomben K. D. Handbook of X-ray Photoelectron Spectroscopy. ULVAC-PHI Inc. (1995).

Mullen C., Irwin A., Pond B. V., Huestis D. L., Coggiola M. J., Oser H. Detection $\underline{\text { of explosives and explosives-related compounds by single photon laser }}$ ionization time-of-flight mass spectrometry. Anal. Chem. 78 (2006): $3807-3814$.

National Police Dog Foundation (2014) accessed on March 2016. < http://www.nationalpolicedogfoundation.org/\#!faqs/cso7>

Primera-Pedrozo O. M., Soto-Feliciano Y. M., Pacheco-Londoño L. C., Hernández-Rivera S. P. Vibrational Spectroscopy Standoff Detection of Explosives. Sens. Imaging 10 (2009): 1-13.

Ryder A. G. Surface enhanced Raman scattering for narcotic detection and applications to chemical biology. Current opinion in chemical biology 9.5 (2005): 489-93. 
Sanchez J., Dipasquale A., Mrse A., and Trogler W. Lewis Acid-Base Interactions Enhance Explosives Sensing in Silacycle Polymers. Anal Bioanal Chem Analytical and Bioanalytical Chemistry 395.2 (2009): 387-392.

Sarah Caygill J., Davis F., and P J Higson S. Current trends in explosive detection techniques. Talanta 88 (2012): 14-29.

Schramm E., Hölzer J., Pütz M., Schulte-Ladbeck R., Schultze R., Sklorz M., Ulrich A., Wieser J., Zimmermann R. Real-time trace detection of $\underline{\text { security-relevant compounds in complex sample matrices by thermal }}$ desorption-single photon ionization-ion trap mass spectrometry (TD-SPIITMS). Anal. Bioanal. Chem. 395 (2009):1795-1807.

Senesac L. and Thundat T. G. Nanosensors for trace explosive detection. Materials Today 11.3 (2008): 28-36.

Shaik U. P. and Ghanashyam Krishna M. Single step formation of indium and tin doped $\mathrm{ZnO}$ nanowires by thermal oxidation of indium-zinc and tin-zinc metal films: Growth and optical properties Ceramics International 40.8 (2014): 13611-13620.

Shen X., Song F., Yang X., Wang Z., Jing M., Wang Y., Hexaferrite/ $\alpha$-iron composite nanowires: Microstructure, exchange-coupling interaction and microwave absorption. Journal of Alloys and Compounds 621 (2015): $146-153$

Shriver-Lake L. C., Patterson C. H. and van Bergen S. K. New Horizons:

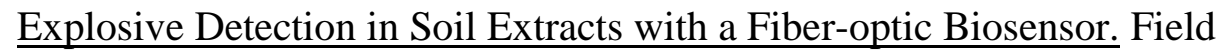
Anal. Chem. Technol 4 (2000): 239. 
Singh S. Sensors--an effective approach for the detection of explosives. Journal Of hazardous materials 144.1-2 (2007): 15-28.

Strle D., Stefane B., Zupanic E., Trifkovic M., Macek M., Jaksa G., Kvasic I., Musevic I., Sensitivity Comparison of Vapor Trace Detection of Explosives Based on Chemo-Mechanical Sensing with Optical Detection and Capacitive Sensing with Electronic Detection. Sensors 14 (2014): $11467-11491$

Sysoev V. V., Schneider T., Goschnick J., Kiselev I., Habicht W., Hahn H., Strelcov E., Kolmakov A., Percolating $\mathrm{SnO}_{2}$ nanowire network as a stable gas sensor: Direct comparison of long-term performance versus $\mathrm{SnO}_{2}$ nanoparticle films. Sensors and Actuators B vol. 139 (2009): 699-703

Tabrizchi M. and ILbeigi V. Detection of Explosives by Positive Corona Discharge Ion Mobility Spectrometry. Journal of Hazardous Materials 176 (2010): 692-696.

Takáts Z., Wiseman J. M., Gologan B., Cooks R. G. Mass Spectrometry Sampling under Ambient Conditions with Desorption Electrospray Ionization. Science 306 (2004): 471-473.

Tharsika $\mathrm{T}$. Gas sensing properties of zinc stannate $(\mathrm{Zn} 2 \mathrm{SnO} 4)$ nanowires prepared by carbon assisted thermal evaporation process. Journal of Alloys and Compounds 618 (2015): 455-462.

Thiesan L., Hannum D., Murray D. W. and Parmeter J. E. Survey of Commercially Available Explosives Detection Technologies and Equipment 2004. Feb. 2005. 
Toal S. and W. Trogler. Polymer Sensors for Nitroaromatic Explosives Detection. Journal of Materials Chemistry J. Mater. Chem. 16.28. (2006): 2871.

Wells K. and Bradley D. A. A review of X-ray explosives detection techniques for checked baggage. Applied radiation and isotopes: including data, instrumentation and methods for use in agriculture, industry and medicine 70.8 (2012): 1729-46.

Wilson, Clay. Improvised Explosive Devices (IEDs) in Iraq and Afghanistan: Effects and Countermeasures. (2007): 1-3. Federation of American Scientists, 21 Nov. 2007. Web. 3 Sept. 2014.

Woolard D. L., Brown E. R., Pepper M., Kemp M. Terahertz Frequency Sensing and Imaging: A Time of Reckoning Future Applications? Proceedings of IEEE 93 (2005): 1722-1743.

Yajun J. One-step method for growing of large scale $\mathrm{ZnO}$ nanowires on zinc foil. Materials Letters 138 (2015): 92-95.

Zimmermann S., Abel N., Baether W., Barth S. An Ion-focusing Aspiration Condenser as an Ion Mobility Spectrometer. Sens. Actuators B: Chem 125 (2007): 428-434. 